\title{
Chiral spin resonance and spin-Hall conductivity in the presence of the electron-electron interactions
}

\author{
A. Shekhter, M. Khodas and A.M. Finkel'stein \\ Department of Condensed Matter Physics, the Weizmann Institute of Science, Rehovot, 76100, Israel
}

\begin{abstract}
We discuss the electron spin resonance in two-dimensional electron gas at zero external magnetic field. This spin-resonance is due to the transitions between the electron states, which are split by the spin-orbit (SO) interaction, and is termed as the chiral spin resonance (CSR). It can be excited by the in-plane component of the electric field of microwave radiation. We show that there exists an inherent relationship between the spin-Hall conductivity and the CSR in a system with the SO interaction. Since in the presence of the SO interaction spin is not conserved, the electron-electron interaction renormalizes the spin-Hall conductivity as well as the frequency of the CSR. The effects of the electron interaction in systems with the SO interaction are analyzed both phenomenologically and microscopically.
\end{abstract}

PACS numbers: 73.21.-b,76.20.+q,73.50.Pz,71.70.Ej

\section{INTRODUCTION}

In systems with spin-orbit ( $\mathrm{SO}$ ) interactions the spin of electrons can be coupled to an electric field, making it possible to manipulate electron spins without applying magnetic fields. This is the main reason why the properties of the electron gas in the presence of the $\mathrm{SO}$ interaction are in the focus of the research in spintronics. $\frac{1}{1}$

In semiconductors with a zinc-blende or a wurtzite lattice the SO interaction originates from the bulk-inversion asymmetry (BIA) of the crystal structure ${ }^{2.3}$, whereas the structure inversion asymmetry (SIA) typical for heterostructures is another source of the SO interaction ${ }^{4.5}$ in two-dimensional electron gas (2DEG).

In the presence of the SO interaction the spin degeneracy of the electron spectrum is lifted. In this context, the possibility of the existence of the spin-Hall current mediated by the SO interaction has been discussed recently 6.7 .8 .9 .10 .11 It is now widely accepted 12.13 .14 .15 .16 that in the static limit the disorder suppresses the spinHall conductivity in the bulk of a macroscopic system. ${ }^{17}$ Therefore, to investigate the bulk effects related to the SO interaction it is worthwhile to turn to the highfrequency phenomena when $\omega \tau \gg 1$.

In this paper we study the ac spin-Hall conductivity in a 2DEG with the Bychkov-Rashba SO interaction. 5 We demonstrate that similar to the Hall conductivity, which in the absence of the SO interaction is inherently related to the cyclotron resonance, the spin-Hall conductivity is related to a specific for $\mathrm{SO}$ systems version of the electron spin resonance (ESR) which has been termed by Rashba as a "combined resonance" $18,19,20$ The combined resonance occurs as a result of the transitions between the electron states, which are split by the combined action of the SO interaction and the Zeeman interaction induced by a static magnetic field. In a 2DEG with the BychkovRashba SO interaction ${ }^{5}$ the spin-split eigenstates (in the limit of zero magnetic field) are characterized by their chirality. We will be interested in the particular limit of the combined resonance when a static magnetic field is ab- sent and the resonance is due to the transitions between electron states with different chirality. To underline the nature of this resonance, we use the term "chiral spin resonance" (CSR), which emphasizes that the discussed electron-spin resonance occurs between the chiral states that are spin split by the SO interaction rather than by the external magnetic field.

In the presence of the SO interaction the dynamics of the total current and the total spin is affected by the electron-electron $(e-e)$ interaction. Consequently, the frequency and the width of the CSR as well as the spin-Hall conductivity acquire renormalization corrections. We start this paper by applying the Kohn's theorem ${ }^{21}$ procedure to analyze the transverse transport coefficients in systems with the SO interaction. In systems with no SO interactions it is easy to show that the absence of the $e-e$ renormalization of the Hall coefficient $R_{H}$ at $\omega_{c} \tau \gg 1$ is a direct consequence of the Kohn's theorem. We observe, however, that the $\mathrm{SO}$ and the $e$-e interactions are not compatible in a sense that the equations of motion for the current operators can be closed when only one of these interactions is present. Still, this approach proves to be useful in finding a relation between the spin-Hall conductivity and the dynamic spin-susceptibility that holds in the clean limit $(\omega \tau \gg 1)$ even in the presence of both the $\mathrm{SO}$ and the $e-e$ interactions ${ }^{22}$

A discussion of the Hall and spin-Hall conductivities following the lines of the Kohn's theorem argumentation is given in Sec. [I] A calculation of the spin-Hall conductivity in the absence of the $e$-e interaction using the equation of motion for the current operators is given in Appendix A (A reader not familiar with the spin-Hall conductivity is recommended to look at the calculations in the Appendix Abefore proceeding further.) In Sec. III we consider the renormalization effects in the dynamic spin-susceptibility induced by the $e$-e interaction within the framework of the phenomenological Fermi-liquid theory. We find the spectrum of the spin excitations in the SO system and, in particular, determine the frequency of the spin resonance. Simultaneously, we calculate the ef- 
fects of the $e-e$ renormalization on the spin-Hall conductivity. This is how the relationship between the spin-Hall conductivity and the CSR can be established. In Sec. IV an alternative microscopic Fermi-liquid analysis of the dynamic spin susceptibility is presented for a justification of the both approaches. In Sec. $\nabla$ we find the disorderinduced width of the CSR including its $e$-e renormalizations. In addition, the Fermi-liquid renormalizations of the D'yakonov-Perel rate of the spin relaxation ${ }^{23}$ are obtained. In the end of this Section we discuss the electrondipole mechanism ${ }^{20}$ of the excitation of the CSR.

Finally, in the concluding section [V] we discuss the perspectives of the experimental observation of the CSR, i.e., the combined resonance in the vanishing magnetic field. To observe the CSR, the spin splitting induced by the SO interaction should be sufficiently isotropic. For the purpose of definiteness, the calculation has been performed for the case of Bychkov-Rashba SO interaction (SIA). However, the results of this analysis are applicable in various other situations. In Appendix B we discuss the forms of the SO interaction due to the lack of the inversion symmetry of the host crystal (BIA $)^{2}$ corresponding to quantum wells grown in different crystallographic directions. We demonstrate that there is a duality transformation relating the linear terms in the SO interaction originating from the SIA and BIA mechanisms. Because of this duality all the conclusions about the spin-Hall conductivity and the electron-spin resonance found for the Bychkov-Rashba SO interaction hold equally well for the linear terms originating from the Dresselhaus SO interaction in the cases of [001]- and [111]-grown quantum wells.

\section{TRANSVERSE CONDUCTIVITIES IN THE PRESENCE OF THE ELECTRON-ELECTRON INTERACTION}

Let us start with the application of the Kohn's theorem procedure to the Hall conductivity (and the Hall coefficient) in a system without a SO interaction. In the presence of a magnetic field $B$ with the corresponding vector potential A a many-electron system is described by the Hamiltonian

$$
H=\sum_{i} \frac{1}{2 m}\left[\mathbf{p}_{i}-\frac{e}{c} \mathbf{A}\left(\mathbf{r}_{i}\right)\right]^{2}+\frac{1}{2} \sum_{i \neq j} V_{e-e}\left(\mathbf{r}_{i}-\mathbf{r}_{j}\right) .
$$

In this paper $m$ denotes the band-structure mass of an electron in a heterostructure in contrast to $m_{e}$ denoting the vacuum mass of the electron. The current operator in the presence of the vector potential $\mathbf{A}$ is

$$
\mathbf{J}=\sum_{i} \frac{1}{m}\left[\mathbf{p}_{i}-\frac{e}{c} \mathbf{A}\left(\mathbf{r}_{i}\right)\right]
$$

It is convenient to introduce "the angular-momentum components" of the current operator $\mathbf{J}$

$$
J^{ \pm}=J^{x} \pm i J^{y}
$$

with the commutator

$$
\left[J^{+}, J^{-}\right]=-2 \frac{\omega_{c}}{m} \hat{N}
$$

where $\omega_{c}=|e| B / m c$ is the frequency of the cyclotron resonance and $\hat{N}$ is an operator of the total number of particles in the system. The Kohn's theorem states that the $e$-e interaction does not change the frequency of the cyclotron resonance ${ }^{21}$ The essence of the theorem is the observation that the electron interaction does not affect the equation of motion for the total current operator

$$
-i \frac{\partial}{\partial t} J^{ \pm}(t)= \pm \omega_{c} J^{ \pm}(t)
$$

The unique property of the operators $J^{ \pm}$is that they connect the pairs of states $l, m$ with the energy difference $E_{l}-E_{m}= \pm \omega_{c}$ only. The closed equation (5) yields for the time dependence of the total current operators

$$
J^{ \pm}(t)=e^{ \pm i \omega_{c} t} J^{ \pm}
$$

With the Kohn's result for the time dependence of the current operators we are fully equipped for the calculation of the conductivity tensor. According to the Kubo formula, the conductivity tensor in the presence of the $e-e$ interaction is given by

$$
\begin{aligned}
\sigma_{+-} & =\frac{e^{2}}{\omega} \int_{0}^{\infty} d t e^{i \omega t}\left\langle\left[J^{+}(t), J^{-}(0)\right]\right\rangle= \\
& =-2 i \frac{n e^{2}}{m \omega} \frac{\omega_{c}}{\omega+\omega_{c}},
\end{aligned}
$$

where $\langle\cdots\rangle$ means quantum-mechanical as well as thermal average and $n$ is the density of the electron gas. Finally, having in mind that $\sigma_{-+}(\omega)=-\sigma_{+-}(-\omega)$, $\sigma_{++}=\sigma_{--}=0$, and $\sigma_{x y}=-\sigma_{y x}=(1 / 4 i)\left[\sigma_{-+}-\sigma_{+-}\right]$, one gets for the transverse components of the conductivity tensor the following result:

$$
\sigma_{x y}=-\frac{n e^{2}}{m} \frac{\omega_{c}}{\omega_{c}^{2}-\omega^{2}} .
$$

Remarkably, the factor $n$ preserves here its physical meaning of the density of the electron gas and does not acquire any renormalization correction in the presence of the electron interaction because of the universal form of the commutator (4). Together with the absence of the renormalization corrections to the cyclotron frequency this leads to an important consequence for the Hall coefficient $R_{H}=\rho_{x y} / B$. Inverting the conductivity tensor one obtains in the dc limit $\omega \rightarrow 0$,

$$
\rho_{x y}=\frac{m}{n e^{2}} \omega_{c} ; R_{H}=-1 / n e c .
$$

Thus, the absence of the renormalization corrections to $R_{H}$ in the clean limit, $\omega_{c} \tau \gg 1$, is a direct consequence of the Kohn's theorem. For the limit of a weak magnetic 
field, $\omega_{c} \tau \ll 1$, the proof of the absence of the renormalization corrections to the Hall coefficient of an interacting electron gas requires a considerable effort ${ }^{24}$

Let us check the possibility to extend the Kohn's theorem to a 2DEG with the Bychkov-Rashba SO interaction $^{5}$ originating from the structure-inversion asymmetry of the heterojunction

$$
H^{S O}=\sum \alpha\left[\boldsymbol{p}_{i} \times \boldsymbol{\ell}\right] \cdot \boldsymbol{\sigma}_{i},
$$

where the unit vector $\boldsymbol{\ell}$ is perpendicular to the plane of the 2DEG. In the presence of the SO interaction the current operator $\mathbf{J}$ contains a spin-dependent term

$$
\mathbf{J}=\sum\left(\frac{\boldsymbol{p}_{i}}{m}+\alpha\left[\boldsymbol{\ell} \times \boldsymbol{\sigma}_{i}\right]\right) \equiv \mathbf{P} / m+2 \alpha[\boldsymbol{\ell} \times \mathbf{S}] .
$$

Here $\mathbf{P}$ and $\mathbf{S}$ are the operators of the total momentum and spin, respectively. Since the $e-e$ interaction commutes with the current operator the interaction drops out from the equation of motion for $\mathbf{J}$, as it takes place in the Kohn's theorem. Still, the current operator has a complicated dynamics due to the SO interaction. For example, for the component $J^{y}$ one gets

$$
i d J^{y} / d t=-2 i \alpha^{2} \sum p_{i}^{x} \sigma_{i}^{z}=-4 i m \alpha^{2} \mathfrak{J}_{z}^{x},
$$

where $\mathfrak{J}_{z}^{x}$ is the $x$ component of $z$-spin current operator

$$
\mathfrak{J}_{z}^{x}=\frac{1}{2} \sum \frac{p_{i}^{x}}{m} \sigma_{i}^{z}
$$

An attempt to get a closed system of equations by supplementing Eq. (12) with the equation of motion for $\mathfrak{J}_{z}^{x}$ fails. It happens in the following way: in the equation of motion for the total current $J^{\alpha}$ the contributions from the $e$-e interaction term $V_{e-e}$ cancel pairwise: $\partial V_{e-e}\left(r_{i}-r_{j}\right) / \partial r_{i}+\partial V_{e-e}\left(r_{i}-r_{j}\right) / \partial r_{j}=0$. On the contrary, in the equation of motion for the spin current $\mathfrak{J}_{z}^{x}$ each of the derivatives is multiplied by a spin operator of different particles and, as a result, the $e$-e interaction does not drop out: $\sigma_{i}^{z} \partial V_{e-e}\left(r_{i}-r_{j}\right) / \partial r_{i}+\sigma_{j}^{z} \partial V_{e-e}\left(r_{i}-\right.$ $\left.r_{j}\right) / \partial r_{j}=\left(\sigma_{i}^{z}-\sigma_{j}^{z}\right) \partial V_{e-e}\left(r_{i}-r_{j}\right) / \partial x_{i} \neq 0$.

The very fact that $V_{e-e}$ does not drop out from the equations of motion indicates that in the presence of the SO interaction the dynamics of the electron gas is affected by the $e$-e interaction. In spite of this complication, the Kohn's theorem approach is useful for proving the relation between the spin-Hall conductivity and the dynamic spin susceptibility that remains intact even in the presence of the $e$ - $e$ interaction (see also Ref. 22). The spin-Hall conductivity $\varsigma_{x y}^{z}$ describes the response of the spin- $z$-component current in the $x$ direction $\mathfrak{J}_{z}^{x}$ to the electric field applied in the $y$ direction. It is determined by the Kubo formula as follows:

$$
\varsigma_{x y}^{z}=\frac{e}{\omega} \int_{0}^{\infty} d t e^{i \omega t}\left\langle\left[\mathfrak{J}_{z}^{x}, J^{y}(-t)\right]\right\rangle .
$$

To explore its relation with the spin susceptibility we eliminate $\mathfrak{J}_{z}^{x}$ in favor of $J^{y}$ with the use of Eq. (12). Performing the time integration by parts one obtains

$$
\varsigma_{x y}^{z}=\frac{i e}{4 m \alpha^{2}} \int_{0}^{\infty} d t e^{i \omega t}\left\langle\left[J^{y}(t), J^{y}(0)\right]\right\rangle .
$$

In a translation-invariant system the total momentum $\mathbf{P}(t)$ is a conserved quantity, the commutator $[\mathbf{P}(t), \mathbf{S}(0)]=[\mathbf{P}(0), \mathbf{S}(0)]=0$, and, therefore, the momentum operator $P^{y}$ drops out from Eq. (15). Finally, one gets

$$
\varsigma_{x y}^{z}(\omega)=\frac{i e}{m} \int_{0}^{\infty} d t e^{i \omega t}\left\langle\left[S^{x}(t), S^{x}(0)\right\rangle .\right.
$$

Thus, there is a direct connection between $\varsigma_{x y}^{z}$ and the dynamic (retarded) spin susceptibility

$$
\begin{aligned}
\varsigma_{x y}^{z} & =\frac{e}{m} \chi_{x x}(q=0, \omega) \\
\chi_{x x}(q=0, \omega) & =\frac{i}{4} \int_{0}^{\infty} d t e^{i \omega t}\left\langle\sum\left[\sigma_{i}^{x}(t), \sigma_{i}^{x}(0)\right]\right\rangle .
\end{aligned}
$$

In the presence of the SO interaction $\chi_{x x}$ has a behavior that differs radically from that when $\alpha=0$. In the absence of the SO interaction the total spin is conserved. Therefore, $\chi_{x x}(q=0, \omega)_{\alpha=0}=0$ and, consequently, the spin-Hall conductivity vanishes at $\alpha=0$, whereas at any finite $\alpha$ one gets $\chi_{x x}(q=0, \omega) \neq 0$ and, consequently, $\varsigma_{x y}^{z} \neq 0$.

It is worth noting that Eq. (17) is valid only in the absence of disorder. The correlation function $\chi_{x x}$, by itself, is insensitive to disorder as long as the elastic scattering rate is less than the spin splitting energy. However, the relation between $\varsigma_{x y}^{z}$ and $\chi_{x x}$ is very subtle $\frac{12.13 .14 .15 .16}{}$, because in the presence of disorder the momentum is not conserved, and there is a competition between the spin and momentum contributions to the current vertex (inter- and intrabranch contributions in terminology of Ref. 16). The involvement of the momentum part of the current operator makes Eq. (17) unapplicable for analyzing the static limit of the spin-Hall conductivity in the presence of disorder or an external magnetic field. Still, Eq. (17) is valid when $\omega \gg \eta_{2}$ [see Eq. (57) and the discussion in the end of Sec. $\nabla$ and will be used for the analysis of the CSR.

\section{FERMI-LIQUID ANALYSIS OF SPIN CORRELATION FUNCTION IN THE PRESENCE OF SO INTERACTION. THE SPIN RESONANCE}

It has been demonstrated above that in the presence of the SO interaction, the dynamics of the total current is affected by the $e-e$ interaction. As a consequence of this fact, the spin-Hall conductivity acquires corrections, which we analyze now with the use of the methods of the phenomenological Fermi-liquid theory. Since the calculation of the spin-Hall conductivity reduces in the clean 
limit to determining the dynamic spin-susceptibility, one can follow the derivation of the spin-waves spectrum in the Fermi liquid in an external magnetic field (see Chap. $1, \S 5$ in Ref. 25. There is an important difference, however, between the spin splitting induced by the external magnetic field and the SO interaction. As a result of the SO interaction the spin of an electron feels an "individual" magnetic field, which is directed perpendicular to the momentum of the electron. For this reason, to analyze the spin dynamics in the presence of the SO interaction it is convenient to introduce the chiral basis with the rotated Pauli matrices $\tau_{\mathbf{p}}^{\nu}=\left(\mathbf{a}_{\mathbf{p}}^{\nu} \cdot \boldsymbol{\sigma}\right)$, where $\mathbf{a}_{\mathbf{p}}^{\nu}=\left\{\mathbf{a}^{1}, \mathbf{a}^{2}, \mathbf{a}^{3}\right\}=\{-\boldsymbol{\ell}, \hat{\mathbf{p}}, \hat{\mathbf{p}} \times \boldsymbol{\ell}\}$ and $\hat{\mathbf{p}}$ stands for a unit vector in the direction of momentum $\mathbf{p}$. [Here we consider the Bychkov-Rashba SO interaction. Similar analysis can be done for the case of SO interaction induced by $\mathrm{BIA}^{2}$ (see Appendix B for details).]

Since $\mathbf{a}_{\mathbf{p}}^{\nu}$ form an orthonormal basis, $\tau$ matrices have the same commutation relations as the Pauli matrices. In the chiral basis, the free single-particle Hamiltonian acquires the diagonal form

$$
H^{S O}=\frac{\mathbf{p}^{2}}{2 m}+\alpha|\mathbf{p}| \tau_{\mathbf{p}}^{3}
$$

with the energy spectrum split into two chiral branches

$$
\epsilon_{p}^{ \pm}=p^{2} / 2 m \pm \alpha p .
$$

In the presence of the $e$ - $e$ interaction the spin splitting induced by the SO interaction is renormalized. It can be determined by a self-consistent equation

$$
\delta \hat{\epsilon}_{\mathbf{p}}^{S O}=\alpha[\mathbf{p} \times \boldsymbol{\ell}] \cdot \boldsymbol{\sigma}+\operatorname{Tr}^{\prime} \int d \Omega^{\prime} \hat{f}_{\mathbf{p} \mathbf{p}^{\prime}} \frac{\partial n}{\partial \epsilon} \delta \hat{\epsilon}_{\mathbf{p}^{\prime}}^{S O} .
$$

where $\hat{f}_{\mathbf{p p}^{\prime}}$ is the function introduced by Landau to describe the effects of electron interaction in the Fermi liquid, and $\operatorname{Tr}^{\prime}$ denotes the trace with respect to the spin indices. In Eq. (20) $\delta \hat{n}_{\mathbf{p}}^{S O}=\partial n / \partial \epsilon \delta \hat{\epsilon}_{\mathbf{p}}^{S O}$ is the response of the distribution function of the quasiparticles to the SO-interaction term, while the integral term describes the modification of the quasiparticle energy spectrum as a result of the change of the quasiparticle distribution. Note that Eq. (20) is a matrix equation in spin space, and we use for the function $\hat{f}_{\mathbf{p p}^{\prime}}$ the standard notation $\nu\left(\epsilon_{F}\right) \hat{f}_{\mathbf{p p}^{\prime}}=F\left(\theta_{\mathbf{p p}^{\prime}}\right)+G\left(\theta_{\mathbf{p p}^{\prime}}\right) \overrightarrow{\boldsymbol{\sigma}} \cdot \overrightarrow{\boldsymbol{\sigma}}^{\prime}$, where $\theta_{\mathbf{p} \mathbf{p}^{\prime}}$ is an angle between $\mathbf{p}$ and $\mathbf{p}^{\prime}$, and $\nu\left(\epsilon_{F}\right)=m^{*} / \pi$ is the renormalized density of states for both spin components in a 2DEG. (Naturally, only the spin-dependent part of the Landau's function is important for the phenomena related to the SO interaction.) To solve Eq. (20) one should expand $G(\theta)$ in a series of $2 D$-harmonics, $G(\theta)=\sum_{m} G^{m} e^{i m \theta}$, and exploit the following property of the Pauli matrices: $\overrightarrow{\boldsymbol{\sigma}} \cdot \operatorname{Tr}\left(\overrightarrow{\boldsymbol{\sigma}}^{\prime} \tau_{\mathbf{p}}^{\nu}\right)=2 \tau_{\mathbf{p}}^{\nu}$. As a result, the renormalized spin splitting of the electron energy spectrum, $\delta \hat{\epsilon}_{\mathbf{p}}^{S O} \equiv \alpha^{*} p_{F} \tau_{\mathbf{p}}^{3}$, is determined by the renormalized SO parameter $\alpha^{*}=\alpha /\left(1+G^{1}\right)$; see also Ref. 26.
To find the dynamic spin susceptibility $\chi(q=0, \omega)$ we calculate a response linear in the time-dependent in-plane magnetic field $B_{x} e^{i \omega t}$. Consider the equation of motion of the density matrix $\delta \hat{n}$ in the Landau's Fermi liquid in the presence of the SO interaction and the perturbation term $\delta \hat{\epsilon}^{B}$, which is introduced by the magnetic field,

$$
\delta \hat{\epsilon}^{B}=-g \mu_{B}\left(\sigma^{x} / 2\right) B_{x} e^{i \omega t}=-\sigma^{x} \mathfrak{F} e^{i \omega t},
$$

where $\mu_{B}=e \hbar / 2 m_{e} c$ and the Lande-factor $g$ depends on the semiconductor. [In GaAs $g=-0.44$, whereas in $I n_{x} G a_{1-x} A s$ heterostructures the absolute value $|g|$ can be an order of magnitude larger]. Since spin variables are involved, $\delta \hat{n}$ is a matrix in spin space and its time evolution is given by the commutator

$$
i \frac{\partial}{\partial t} \delta \hat{n}_{\mathbf{p}}=\left[\delta \hat{n}_{\mathbf{p}}, \delta \hat{\epsilon}_{\mathbf{p}}\right]
$$

In our case $\delta \hat{\epsilon}_{\mathbf{p}}=\alpha p_{F} \tau_{\mathbf{p}}^{3}+\delta \hat{\epsilon}^{B}+\operatorname{Tr}^{\prime} \int d \Omega^{\prime} \hat{f}_{\mathbf{p p}^{\prime}} \delta \hat{n}_{\mathbf{p}^{\prime}}$, where the last term accounts for the effects of the Fermi liquid.

To find the response linear in the magnetic field, one has to consider the case when the magnetic term is much smaller than the spin-orbit one, $\delta \hat{\epsilon}^{B} \ll 2 \alpha p_{F}$. In equation (22) the static part of $\delta \hat{n}_{\mathbf{p}}$ induced by the SO interaction, $\delta \hat{n}_{\mathbf{p}}^{S O}$, should be separated from a time-dependent part $\delta \hat{n}_{\mathbf{p}}^{B}$

$$
\delta \hat{n}_{\mathbf{p}}=\delta \hat{n}_{\mathbf{p}}^{S O}+\delta \hat{n}_{\mathbf{p}}^{B}
$$

After this separation Eq. (22) can be linearized with respect to $\delta \hat{\epsilon}^{B}$ and $\delta \hat{n}_{\mathbf{p}}^{B}$

$$
\begin{aligned}
i \frac{\partial}{\partial t} \hat{u}_{\mathbf{p}} & =-\left[\delta \hat{\epsilon}_{\mathbf{p}}^{S O}, \hat{u}_{\mathbf{p}}+\nu\left(\epsilon_{F}\right) \frac{1}{2} \operatorname{Tr}^{\prime} \int d \Omega^{\prime} \hat{f}_{\mathbf{p p}^{\prime}} \hat{u}_{\mathbf{p}^{\prime}}\right] \\
& +\left[\delta \hat{\epsilon}_{\mathbf{p}}^{S O}, \sigma_{x}\right] \mathfrak{F} e^{i \omega t}
\end{aligned}
$$

Here we rewrite $\delta \hat{n}_{\mathbf{p}}^{B}$ in terms of the displacement function $\hat{u}_{\mathbf{p}}$, describing the deformation of the Fermi surface, $\delta \hat{n}_{\mathbf{p}}^{B}=(\partial n / \partial \epsilon) \hat{u}_{\mathbf{p}}$ (note that $\hat{u}_{\mathbf{p}}$ depends on the direction of the vector $\mathbf{p}$ and is a matrix in spin space). In Eq. (24) the static part $\delta \hat{n}_{\mathbf{p}}^{S O}$ has been absorbed by $\delta \hat{\epsilon}_{\mathbf{p}}^{S O}$ giving the renormalized spin-splitting energy $\Delta$

$$
\delta \hat{\epsilon}_{\mathbf{p}}^{S O}=\alpha^{*} p_{F} \tau_{\mathbf{p}}^{3}=\frac{1}{2} \Delta \tau_{\mathbf{p}}^{3}
$$

With the use of $\sigma_{x}=\left(p_{x} \tau_{\mathbf{p}}^{2}+p_{y} \tau_{\mathbf{p}}^{3}\right) / p$ the "driving-force" term in the above equation can be rewritten as

$$
\left[\delta \hat{\epsilon}_{\mathbf{p}}^{S O}, \sigma_{x}\right] \mathfrak{F} e^{i \omega t}=-i \Delta \tau^{1} \frac{p_{x}}{p} \mathfrak{F} e^{i \omega t}
$$

To solve Eq. (24), one have to represent the matrix $\hat{u}_{\mathbf{p}}$ in terms of $\tau$ matrices: $\hat{u}_{\mathbf{p}}=u_{1}\left(\theta_{\mathbf{p}}\right) \tau^{1}+u_{2}\left(\theta_{\mathbf{p}}\right) \tau_{\mathbf{p}}^{2}+$ $u_{3}\left(\theta_{\mathbf{p}}\right) \tau_{\mathbf{p}}^{3}$, where $\theta_{\mathbf{p}}$ denotes the direction of the vector $\mathbf{p}$. The coefficients $u_{i}\left(\theta_{\mathbf{p}}\right)$ are determined by a system of 
equations $\left(\theta_{\mathbf{p} \mathbf{p}^{\prime}} \equiv \theta_{\mathbf{p}}-\theta_{\mathbf{p}^{\prime}}\right)$

$$
\begin{aligned}
\Delta^{-1} \frac{d u_{1}\left(\theta_{\mathbf{p}}\right)}{d t} & =u_{2}\left(\theta_{\mathbf{p}}\right)+\int d \theta_{\mathbf{p}^{\prime}} G\left(\theta_{\mathbf{p} \mathbf{p}^{\prime}}\right) \cos \theta_{\mathbf{p}_{\mathbf{p}^{\prime}}} u_{2}\left(\theta_{\mathbf{p}^{\prime}}\right) \\
& -\mathfrak{F} e^{i \omega t} \cos \theta_{\mathbf{p}} \\
\Delta^{-1} \frac{d u_{2}\left(\theta_{\mathbf{p}}\right)}{d t} & =-u_{1}\left(\theta_{\mathbf{p}}\right)-\int d \theta_{\mathbf{p}^{\prime}} G\left(\theta_{\mathbf{p} \mathbf{p}^{\prime}}\right) u_{1}\left(\theta_{\mathbf{p}^{\prime}}\right) \\
\frac{d u_{3}\left(\theta_{\mathbf{p}}\right)}{d t} & =0 .
\end{aligned}
$$

In the transition from Eq. (24) to Eqs. 27a and 27b it has been used that $\overrightarrow{\boldsymbol{\sigma}} \cdot \operatorname{Tr}\left(\overrightarrow{\boldsymbol{\sigma}} \tau_{\mathbf{p}^{\prime}}^{\nu}\right)=2 \tau_{\mathbf{p}^{\prime}}^{\nu}$ and that the commutator $\left[\tau_{\mathbf{p}}^{3}, \tau_{\mathbf{p}^{\prime}}^{2}\right]=-2 i \tau^{1} \cos \theta_{\mathbf{p} \mathbf{p}^{\prime}}$.

Since the function $\hat{f}_{\mathbf{p p}^{\prime}}$ depends on the directions of vectors $\mathbf{p}$ and $\mathbf{p}^{\prime}$ through $\cos \theta_{\mathbf{p p}^{\prime}}$ only, Eqs. (27a)-(27c) can be solved by expanding $u_{i}(\theta)$ in $2 D$ harmonics, $u_{i}(\theta)=\sum_{m} u_{i}^{m} e^{i m \theta}$

$$
\begin{aligned}
\Delta^{-1} \frac{d u_{1}^{m}}{d t}=u_{2}^{m} & {\left[1+\frac{1}{2}\left(G^{m+1}+G^{m-1}\right)\right] } \\
& -\frac{1}{2}\left(\delta_{m, 1}+\delta_{m,-1}\right) \mathfrak{F} e^{i \omega t}, \\
\Delta^{-1} \frac{d u_{2}^{m}}{d t}=- & u_{1}^{m}\left(1+G^{m}\right) .
\end{aligned}
$$

It has been used here that $G(\theta) \cos \theta \rightarrow(1 / 2)\left(G^{m+1}+\right.$ $\left.G^{m-1}\right)$ and that the harmonics coefficients are even in $m, G^{m}=G^{-m}$ (this is because the function $\hat{f}_{\mathbf{p} \mathbf{p}^{\prime}}$ is even in $\left.\theta_{\mathbf{p p}^{\prime}}\right)$. After the time-Fourier transform one gets the frequencies of the spin waves

$$
\omega_{m}^{2}(q=0)=\Delta^{2}\left(1+G^{m}\right)\left[1+\frac{1}{2}\left(G^{m+1}+G^{m-1}\right)\right] .
$$

In the absence of the $e$ - $e$ interaction $\omega_{m}$ does not depend on $m$ as each spin precess independently in the individual field induced by the SO interaction with the same frequency. The $e-e$ interaction couples the precession motion of different spins, thereby lifting the degeneracy of the precession by renormalizing the frequency. As a result one gets a set of the spin-wave excitations corresponding to different $2 D$-harmonics. Unlike the noninteracting case where the spin precession is circular, in the presence of the $e-e$ interaction, the precession is elliptical.

When an electromagnetic field is applied to the electron gas the CSR can be excited. The only harmonics activated by the in-plane field are those with $m= \pm 1$

$$
\begin{aligned}
& u_{1}^{ \pm 1}=-i \frac{\omega \Delta}{2\left(\omega_{1}^{2}-\omega^{2}\right)} \mathfrak{F} e^{i \omega t}, \\
& u_{2}^{ \pm 1}=\left(1+G^{1}\right) \frac{\Delta^{2}}{2\left(\omega_{1}^{2}-\omega^{2}\right)} \mathfrak{F} e^{i \omega t},
\end{aligned}
$$

and, therefore, the CSR frequency $\omega^{C S R}$ is determined by $\omega_{1}$

$$
\omega^{C S R}=\Delta\left\{\left(1+G^{1}\right)\left[1+\frac{1}{2}\left(G^{0}+G^{2}\right)\right]\right\}^{1 / 2} .
$$

Unlike the ESR in the absence of the SO where the resonance frequency is not renormalized, $\omega^{C S R}$ is renormalized by the $e-e$ interaction. This is quite natural as the ESR analog of the Kohn's theorem ${ }^{21}$ does not hold in the presence of the SO interaction.

To find the spin-spin correlation function $\chi_{x x}$ we calculate $S_{x}=\frac{1}{2} \nu\left(\epsilon_{F}\right) \operatorname{Tr} \int \hat{u}_{\mathbf{p}}\left(\sigma^{x} / 2\right)(d \theta / 2 \pi)$ as a response of the electron gas to the magnetic field $B_{x}$. Only $\tau_{\mathbf{p}}^{2}$ component of $\hat{u}_{\mathbf{p}}$ contributes and, therefore,

$$
\begin{aligned}
S_{x} & =\frac{1}{4} \nu\left(\epsilon_{F}\right) \sum_{m} u_{2}^{m}\left[\delta_{m, 1}+\delta_{m,-1}\right]= \\
& =\nu\left(\epsilon_{F}\right)\left(1+G^{1}\right) \frac{\Delta^{2}}{8\left[\left(\omega^{C S R}\right)^{2}-\omega^{2}\right]}\left(g \mu_{B}\right) B_{x} .
\end{aligned}
$$

Noting that $\chi_{x x}$ is equal to $S_{x} /\left(g \mu_{B} B_{x}\right)$, one obtains in the limit of small $\omega \ll \omega^{C S R} \sim \Delta$ that

$$
\chi_{x x}(q=0, \omega \rightarrow 0)_{\alpha \neq 0}=\frac{1}{8} \frac{\nu\left(\epsilon_{F}\right)}{1+\frac{1}{2}\left(G^{0}+G^{2}\right)},
$$

and correspondingly in the absence of disorder the renormalized value of the spin-Hall conductivity in the limit of small frequency (see the discussion in the end of Section II) is equal to

$$
\varsigma_{x y}^{z}=\frac{e}{8 \pi} \frac{1}{1+\frac{1}{2}\left(G^{0}+G^{2}\right)} \frac{m^{*}}{m} .
$$

The angular structure of the corrections to $\varsigma_{x y}^{z}$ calculated in Ref. 22 to the lowest order in the $e$-e interaction are in agreement with this result.

In the above consideration we find the dynamic spin susceptibility by calculating the response to a magnetic field $B e^{i \omega t}$ coupled to the magnetic moments of electrons via the Zeeman interaction. Actually, as a mechanism of the excitation of the CSR this type of coupling is very ineffective. In the presence of the SO interaction the electromagnetic field A can excite the spin-flip transitions much more effectively by coupling through the electric-dipole interaction $-(e / c)$ AJ. (For the electricdipole excited spin resonance see Ref. 20). The relative effectiveness of the two mechanisms is of the order of the ratio of the Compton length to the electron wavelength: $(\lambda / \lambda)^{2} \sim 10^{-9}-10^{-8}$. We postpone the discussion of excitation of the CSR as well as of the width of the resonance to Sec. D

Finally, a further comment is in order. The above calculation demonstrates an inherent relationship between the spin-Hall conductivity and the CSR. The same correlation function, $\chi_{x x}(q=0, \omega)_{\alpha \neq 0}$, describes the resonance and determines the value of $\varsigma_{x y}^{z}$, including its static limit. Actually, the existence of a relationship between a transverse conductivity and a corresponding resonance is generic. In clean systems, in the absence of dissipation, the longitudinal conductivity $\sigma_{x x}(\omega)$ vanishes when the frequency $\omega$ is in the range $1 / \tau \ll \omega \ll \Delta E$ as at such frequencies the dipole transitions with the energy $\Delta E$ cannot be excited. Unlike the longitudinal, the Hall 
conductivity, as well as the spin-Hall one, are related not to the real transitions but to the virtual. This leads to the generic relationship between the transverse conductivities and the corresponding resonance; see also Sec. [II where the connection between the Hall conductivity and the cyclotron resonance has been demonstrated.

\section{SPIN-SUSCEPTIBILITY IN THE PRESENCE OF THE SO INTERACTION: MICROSCOPIC CALCULATION}

In this section we develop a microscopic derivation of the dynamic spin susceptibility as an alternative to the phenomenological description presented in Sec. III As a whole, we follow the scheme elaborated for the microscopic derivation of the dynamic spin susceptibility by one of us in Ref. 27.

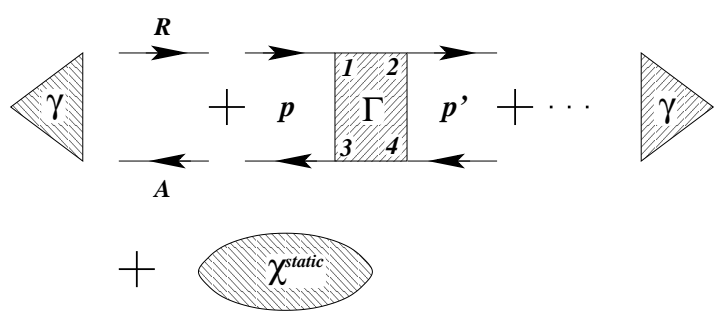

FIG. 1: Spin-density correlation function

Let us discuss the ladder diagrams for the spin-density correlation function presented in Fig. [1 We choose to work with the amplitudes known in the Fermi-liquid theory as $\Gamma^{k}$. This approach has the following reasoning. A two-particle vertex function $\Gamma(\omega, \mathbf{k})$ includes an irreducible part, the contributions from the incoherent scattering, and, most importantly, the contributions from a multiple rescattering of electron-hole quasiparticle pairs. Apart from small corrections $\sim \alpha / v_{F} \ll 1$, neither the irreducible part of the vertex function nor the contributions from the incoherent scattering are sensitive to a small modification of the electron spectrum because they accumulate their values far from the Fermi surface. On the contrary, the rescattering of a quasiparticle pair requires certain care. The reason is that the contribution from a cross section with an electron-hole pair as an intermediate state is equal to a singular combination $\mathbf{v}_{F} \mathbf{k} /\left(i \omega_{n}-\mathbf{v}_{F} \mathbf{k}\right)$, where $\mathbf{v}_{F} \mathbf{k}$ originates from $\delta \epsilon_{k}(\mathbf{p})=\epsilon(\mathbf{p}+\mathbf{k})-\epsilon(\mathbf{p})$ (see Chap. 2, $\S 17$ in Ref. 25). Index $k$ in $\Gamma^{k}$ means that in $\Gamma(\omega, \mathbf{k})$ the contributions of such cross sections are taken as follows: one first takes $\omega=0$ and only afterward takes the limit $k \rightarrow 0$. In the presence of the SO interaction the energy difference $\delta \epsilon_{k}(\mathbf{p})$ acquires a gap when the two quasiparticles have different chirality. The order of limits corresponding to $\Gamma^{k}$ makes this amplitude to be not sensitive to a gap in the energy spectrum of the quasiparticles. Indeed, in the considered order of limits the combination $\delta \epsilon_{\mathbf{k}}(\mathbf{p}) /\left[i \omega_{n}-\delta \epsilon_{\mathbf{k}}(\mathbf{p})\right]$ is equal to -1 for any energy spec- trum of electrons. Altogether this argumentation ${ }^{28}$ leads to the conclusion that the values of the static amplitudes $\Gamma^{k}$ are not modified by a $\mathrm{SO}$ interaction apart from small corrections $\sim \alpha / v_{F} \ll 1$. This feature of the amplitude $\Gamma^{k}$ makes it particularly convenient for the purposes of the microscopic analysis. Diagrammatically the amplitude $\Gamma^{k}$ can be defined as a two-particle amplitude irreducible with respect to a $R A$ section (by the $R A$ section we understand a product of the two Green's functions when one of them is retarded, while the other one is advanced). With the use of $\Gamma^{k}$, the ladder diagrams for the two-particle correlation functions are rearranged in such a way that the blocks of the combination $i \omega_{n} /\left[i \omega_{n}-\delta \epsilon_{\mathbf{k}}(\mathbf{p})\right]$ rather than $\delta \epsilon_{\mathbf{k}}(\mathbf{p}) /\left[i \omega_{n}-\delta \epsilon_{\mathbf{k}}(\mathbf{p})\right]$ stand separated by amplitudes $\Gamma^{k}$.

Depending on the spin structure the two-particle amplitude can be split into two parts

$$
\begin{aligned}
& \frac{\nu\left(\epsilon_{F}\right)}{2} a^{2} \hat{\Gamma}_{1 \alpha_{3} \alpha_{4}}^{k \alpha_{1} \alpha_{2}}\left(\mathbf{p}, \mathbf{p}^{\prime}\right)=\Gamma_{1}\left(\mathbf{p}, \mathbf{p}^{\prime}\right) \delta_{\alpha_{1}, \alpha_{3}} \delta_{\alpha_{2}, \alpha_{4}} \\
& \frac{\nu\left(\epsilon_{F}\right)}{2} a^{2} \hat{\Gamma}_{2}^{k} \hat{\alpha}_{\alpha_{3} \alpha_{4}}^{\alpha_{1} \alpha_{2}}\left(\mathbf{p}, \mathbf{p}^{\prime}\right)=-\Gamma_{2}\left(\mathbf{p}, \mathbf{p}^{\prime}\right) \delta_{\alpha_{1}, \alpha_{2}} \delta_{\alpha_{3}, \alpha_{4}} .
\end{aligned}
$$

Here matrices $\hat{\Gamma}_{1,2}^{k}$ denote the spin-dependent amplitudes, while the dimensionless function $\Gamma_{1,2}$ determine the parameters of the Fermi-liquid theory (in $\Gamma_{1,2}$ the index $k$ is omitted). The minus sign in the amplitude $\Gamma_{2}$ is due to the anticommutation of the fermionic operators. The factor $a$ appears in a standard way because it describes the weight of the quasiparticle part in the Green's function ${ }^{25}$ For electrons at the Fermi energy the functions $\Gamma_{1,2}\left(\mathbf{p}, \mathbf{p}^{\prime}\right)=\Gamma_{1,2}\left(\theta_{\mathbf{p p}^{\prime}}\right)$ depend on the scattering angle $\theta_{\mathbf{p p}^{\prime}}$ only. The coefficients of the expansion of $\Gamma_{1,2}\left(\theta_{\mathbf{p p}^{\prime}}\right)$ in angular harmonics are used as the parameters of the Fermi-liquid theory. In $2 D$ they are defined as follows:

$$
\Gamma_{1,2}^{m}=\int \frac{d \theta}{2 \pi} \Gamma_{1,2}(\theta) \exp (-i m \theta)
$$

Now we turn to triangle vertices $\gamma$. Like $\Gamma^{k}$, the vertex

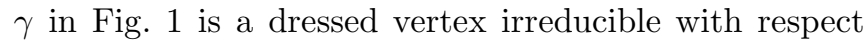
to $R A$ sections (i.e., it extends from an external vertex to the first $R A$ section). The arguments concerning insensitivity of the static limit of the two-particle vertex functions to the $\mathrm{SO}$ interaction remains also valid (with an accuracy $\sim \alpha / v_{F} \ll 1$ ) for the renormalized "triangle" vertex $\gamma$. Since we are interested in the spin-density correlation function, we consider the case when the external vertices contain a spin operator $\sigma_{x} / 2$ (such a vertex is denoted below as $\hat{\gamma}^{\sigma_{x}}$ ). Due to the Fermi-liquid corrections the vertex $\hat{\gamma}^{\sigma_{x}}$ acquires the renormalization factor $\left(1+\Gamma_{2}^{0}\right)$, where $\Gamma_{2}^{0}$ is zero harmonics of the interaction amplitude $\Gamma_{2}\left(\mathbf{p}, \mathbf{p}^{\prime}\right)$. The last contribution in Fig. 10 to be commented on is the static spin susceptibility $\chi_{x x}(\omega=0)$ (this correlation function does not contain any $R A$ sections). According to the same argumentation it is equal to $(1 / 4) \nu\left(\epsilon_{F}\right)\left(1+\Gamma_{2}^{0}\right)$. 
The singular part of the matrix Green's function in the presence of the Bychkov-Rashba SO interaction is

$$
\hat{\mathcal{G}}(i \epsilon, \mathbf{p})=\sum_{\zeta= \pm 1}|\zeta \mathbf{p}\rangle \frac{a}{i \epsilon-\epsilon_{p}^{\zeta}+\mu}\langle\zeta \mathbf{p}|
$$

where the residue $a$ is a weight of the quasiparticle part in the Green's function. [In what follows the singular parts of the Green's functions will be used without the factor $a$. This is the reason for attaching $a^{2}$ to the matrices $\hat{\Gamma}_{1,2}^{k}$ in the relations (35). With the use of the effective mass $m^{*}$ and a proper redefinition of triangle vertices $\gamma$ the explicit dependence on the residue $a$ drops out from the Fermi-liquid calculations ${ }^{25}$ ] The direct product of spinors $|\zeta \mathbf{p}\rangle\langle\zeta \mathbf{p}|$ in the Green's function $\hat{\mathcal{G}}(i \epsilon, \mathbf{p})$ is the projector onto the chiral states with the eigenenergies $\epsilon_{p}^{\zeta}=p^{2} / 2 m^{*}+\zeta \Delta / 2$; here and in what follows the chiral state index $\zeta= \pm 1$. The eigenspinors in Eq. (37) can be found from the eigenvalue problem for $\tau_{\mathbf{p}}^{3}$-matrix

$$
\begin{aligned}
\tau_{\mathbf{p}}^{3}|\zeta \mathbf{p}\rangle & =\zeta|\zeta \mathbf{p}\rangle, \\
|\zeta \mathbf{p}\rangle & =\frac{1}{\sqrt{2}}\left[\begin{array}{c}
i \zeta e^{-i \vartheta_{\mathbf{p}}} \\
1
\end{array}\right]
\end{aligned}
$$

where $e^{ \pm i \vartheta_{\mathbf{p}}}=\left(p_{x} \pm i p_{y}\right) / p$.

To conduct the calculation in the chiral basis, the spinors will be transferred from the Green's functions to the interaction amplitudes and to the vertices $\hat{\gamma}^{\sigma_{x}}$ (see Fig. (1). As a result, one gets for the matrices $\hat{\Gamma}_{1,2}$

$\frac{\nu\left(\epsilon_{F}\right)}{2} a^{2} \hat{\Gamma}_{1}^{k}{ }_{\zeta_{3} \zeta_{4}}^{\zeta_{1} \zeta_{2}}\left(\mathbf{p}, \mathbf{p}^{\prime}\right)=\Gamma_{1}\left(\mathbf{p}, \mathbf{p}^{\prime}\right)\left\langle\zeta_{3} \mathbf{p} \mid \zeta_{1} \mathbf{p}\right\rangle\left\langle\zeta_{2} \mathbf{p}^{\prime} \mid \zeta_{4} \mathbf{p}^{\prime}\right\rangle$,
$\frac{\nu\left(\epsilon_{F}\right)}{2} a^{2} \hat{\Gamma}_{2}^{k}{ }_{2}^{\zeta_{1} \zeta_{3}}\left(\mathbf{p}, \mathbf{p}^{\prime}\right)=-\Gamma_{2}\left(\mathbf{p}, \mathbf{p}^{\prime}\right)\left\langle\zeta_{2} \mathbf{p}^{\prime} \mid \zeta_{1} \mathbf{p}\right\rangle\left\langle\zeta_{3} \mathbf{p} \mid \zeta_{4} \mathbf{p}^{\prime}\right\rangle$,

and, similarly, for the vertices $\hat{\gamma}^{\sigma_{x}}$

$$
\begin{aligned}
& a \hat{\gamma}^{\sigma_{x} \zeta_{1}}(\mathbf{p})=\left(1+\Gamma_{2}^{0}\right)\left\langle\zeta_{1} \mathbf{p}\left|\frac{\sigma_{x}}{2}\right| \zeta_{2} \mathbf{p}\right\rangle \\
& a{ }_{\zeta_{2}}^{\zeta_{1}} \hat{\gamma}^{\sigma_{x}}(\mathbf{p})=\left(1+\Gamma_{2}^{0}\right)\left\langle\zeta_{2} \mathbf{p}\left|\frac{\sigma_{x}}{2}\right| \zeta_{1} \mathbf{p}\right\rangle
\end{aligned}
$$

where the first and second lines correspond to the left and right triangle vertices in Fig. 1

The matrix elements appearing in Eqs. (39) and (40) can be easily found:

$$
\begin{aligned}
\left\langle\zeta \mathbf{p} \mid \zeta^{\prime} \mathbf{p}^{\prime}\right\rangle & =\frac{\zeta \zeta^{\prime} e^{i\left(\vartheta_{\mathbf{p}}-\vartheta_{\mathbf{p}^{\prime}}\right)}+1}{2}, \\
\left\langle\zeta \mathbf{p}\left|\sigma_{x}\right| \zeta^{\prime} \mathbf{p}^{\prime}\right\rangle & =\frac{\zeta e^{i \vartheta_{\mathbf{p}}}-\zeta^{\prime} e^{-i \vartheta_{\mathbf{p}^{\prime}}}}{2 i} .
\end{aligned}
$$

Then, for the matrix $\hat{\Gamma}_{1}$ one has

$$
\frac{\nu\left(\epsilon_{F}\right)}{2} a^{2} \hat{\Gamma}_{1 \zeta_{3} \zeta_{4}}^{k} \zeta_{1} \zeta_{2}\left(\mathbf{p}, \mathbf{p}^{\prime}\right)=\frac{1}{4} \Gamma_{1}\left(\vartheta \mathbf{p p}^{\prime}\right)\left(1+\zeta_{1} \zeta_{3}\right)\left(1+\zeta_{2} \zeta_{4}\right),
$$

and for the matrix $\hat{\Gamma}_{2}$, which is of special importance since it controls the dynamics of spins, one gets $\left(\vartheta_{\mathbf{p p}^{\prime}}=\right.$ $\left.\vartheta_{\mathbf{p}}-\vartheta_{\mathbf{p}^{\prime}}\right)$

$$
\begin{aligned}
& \frac{\nu\left(\epsilon_{F}\right)}{2} a^{2} \hat{\Gamma}_{2}^{k} \zeta_{3} \zeta_{1} \zeta_{2}\left(\mathbf{p}, \mathbf{p}^{\prime}\right)=-\frac{1}{4} \Gamma_{2}\left(\vartheta_{\mathbf{p p}^{\prime}}\right)\left[1+\zeta_{1} \zeta_{2} \zeta_{3} \zeta_{4}+\zeta_{1} \zeta_{2} e^{-i \vartheta_{\mathbf{p p}^{\prime}}}\right. \\
& \left.+\zeta_{3} \zeta_{4} e^{i \vartheta_{\mathbf{p p}^{\prime}}}\right] \text {. }
\end{aligned}
$$

Note that there appears an additional angular dependence because of the factors $e^{ \pm i \vartheta_{\mathbf{p p}^{\prime}}}$ and therefore in the expansion in a series of $2 D$-harmonics one should take into consideration that $\Gamma(\vartheta) e^{ \pm i \vartheta} \rightarrow \Gamma^{m \mp 1}$.

It will be convenient to represent the matrices $\hat{\Gamma}$ in the chiral basis by $4 \times 4$ matrices $\hat{\Gamma}_{i j}$. For that we choose the following convention. The first index $i$ represents the left pair of indices $\left(\begin{array}{l}\zeta_{1} \\ \zeta_{3}\end{array}\right)$ in the order $\left(\begin{array}{l}+ \\ +\end{array}\right),\left(\begin{array}{c}- \\ -\end{array}\right),\left(\begin{array}{c}+ \\ -\end{array}\right),\left(\begin{array}{l}- \\ +\end{array}\right)$, while the second index $j$ represents the right pair of indices $\left(\begin{array}{l}\zeta_{2} \\ \zeta_{4}\end{array}\right)$ in the same order. Finally, after the expansion in $2 D$-harmonics, the explicit block-form expressions for the matrices $\hat{\Gamma}_{i j}$ look as follows $\left(\Sigma_{x}=\left|\begin{array}{ll}0 & 1 \\ 1 & 0\end{array}\right|\right)$ :

$$
\begin{gathered}
\frac{\nu\left(\epsilon_{F}\right)}{2} a^{2} \hat{\Gamma}_{2}^{k}(m)_{i j}= \\
-\frac{\Gamma_{2}^{m}}{2}\left|\begin{array}{cc}
1+\Sigma_{x} & 0 \\
0 & 1+\Sigma_{x}
\end{array}\right|-\frac{\Gamma_{2}^{m+1}}{4}\left|\begin{array}{cc}
1-\Sigma_{x} & 1-\Sigma_{x} \\
1-\Sigma_{x} & 1-\Sigma_{x}
\end{array}\right| \\
\quad-\frac{\Gamma_{2}^{m-1}}{4}\left|\begin{array}{rr}
1-\Sigma_{x} & -1+\Sigma_{x} \\
-1+\Sigma_{x} & 1-\Sigma_{x}
\end{array}\right|
\end{gathered}
$$

and

$$
\frac{\nu\left(\epsilon_{F}\right)}{2} a^{2} \hat{\Gamma}_{1}^{k}(m)_{i j}=\Gamma_{1}^{m}\left|\begin{array}{cc}
1+\Sigma_{x} & 0 \\
0 & 0
\end{array}\right|
$$

Note, that one can combine $\hat{\Gamma}_{1}$ with the top-left block in the first term of $\hat{\Gamma}_{2}$ to create the amplitude $\left(\Gamma_{1}-\right.$ $\left.\Gamma_{2} / 2\right)\left(1+\Sigma_{x}\right)$, which controls the singlet channel of the electron-hole excitations (charge-density excitations). Since the spin dynamics is controlled by $\left(1-\Sigma_{x}\right)$ and $\left(1+\Sigma_{x}\right)\left(1-\Sigma_{x}\right)=0$, the singlet channel amplitude is decoupled from spin excitations.

It remains to calculate the triangle vertices $\hat{\gamma}^{\sigma_{x}}$, which are represented in a four-row-column form as $\left(\gamma^{\sigma_{x}}\right)_{i}$ and ${ }_{j}\left(\gamma^{\sigma_{x}}\right)$. For the left vertex, one has $\left(\gamma^{\sigma_{x}}\right)_{i} \equiv a \hat{\gamma}^{\sigma_{x}} \zeta_{\zeta_{2}}=$ $\left(1+\Gamma_{2}^{0}\right)\left\langle\zeta_{1} \mathbf{p}\left|\sigma_{x} / 2\right| \zeta_{2} \mathbf{p}\right\rangle$ and for the right vertex, ${ }_{j}\left(\gamma^{\sigma_{x}}\right) \equiv$ $a_{\zeta_{2}}^{\zeta_{1}} \hat{\gamma}^{\sigma_{x}}=\left(1+\Gamma_{2}^{0}\right)\left\langle\zeta_{2} \mathbf{p}\left|\sigma_{x} / 2\right| \zeta_{1} \mathbf{p}\right\rangle$. With the use of Eq. (41) one obtains

$$
\begin{aligned}
\left(\gamma^{\sigma_{x}}\right)_{i} & =\frac{\left(1+\Gamma_{2}^{0}\right)}{4 i}\left[\zeta_{1} \delta^{m,-1}-\zeta_{2} \delta^{m, 1}\right] \\
& =\frac{\left(1+\Gamma_{2}^{0}\right)}{4 i}\left(\delta^{m,-1}\left|\begin{array}{c}
\psi \\
\psi
\end{array}\right|+\delta^{m, 1}\left|\begin{array}{c}
-\psi \\
\psi
\end{array}\right|\right)
\end{aligned}
$$

Here the column $\psi=\left[\begin{array}{r}1 \\ -1\end{array}\right]$ has been introduced to 
shorten the notations. Similarly,

$$
\begin{aligned}
j\left(\gamma^{\sigma_{x}}\right) & =-\frac{\left(1+\Gamma_{2}^{0}\right)}{4 i}\left[\zeta_{1} \delta^{m,-1}-\zeta_{2} \delta^{m, 1}\right] \\
& =-\frac{\left(1+\Gamma_{2}^{0}\right)}{4 i}\left(\delta^{m,-1}\left|\begin{array}{c}
\psi \\
\psi
\end{array}\right|+\delta^{m, 1}\left|\begin{array}{c}
-\psi \\
\psi
\end{array}\right|\right) .
\end{aligned}
$$

To proceed further, we discuss a $R A$ section in Fig. 1] Since we are studying the dynamic susceptibility, $\chi_{x x}(q=0, \omega)$, the momenta in the product of the two Green's functions $G^{R} G^{A}$ coincide. After the integration over $\xi=p^{2} / 2 m^{*}-\mu$ the product of two Green's functions yields

$$
\left(G^{R} G^{A}\right)_{\zeta_{2}}^{\zeta_{1}}=\frac{1}{2} \nu\left(\epsilon_{F}\right) \frac{2 \pi i}{i \omega_{n}-\Delta\left(\zeta_{1}-\zeta_{2}\right) / 2} .
$$

Here the product of the Green's functions with different chirality acquires the difference of the frequency and the spin splitting of the energy spectrum in the denominator, while $(G G)_{+}^{+}$and $(G G)_{-}^{-}$are insensitive to the $\mathrm{SO}$ spin splitting. To describe the rescattering of a pair of quasiparticles, one has to consider a ladder of $R A$ sections with the amplitudes $\hat{\Gamma}^{k}$ in between. The amplitudes $\hat{\Gamma}^{k}$ are accompanied by the frequency summation. Ultimately, the geometrical series of the ladder diagrams for the two-particle propagation function yields $\left[\left(G^{R} G^{A}\right)^{-1}-\left(\omega_{n} / 2 \pi\right) \hat{\Gamma}^{k}\right]^{-1}$, where $\hat{\Gamma}^{k}$ is determined in Eqs. (45) and (44), whereas the product $\left(G^{R} G^{A}\right)$ is considered as a matrix with the diagonal elements only that are given by Eq. (48). Owing to the chiral nature of the spectrum of the excitations the triangle vertices $\hat{\gamma}^{\sigma_{x}}$ activate the channels with $m= \pm 1$ only. This fact has been already observed in the phenomenological treatment and here reveals itself in Eqs. (46) and (47) through the Kronecker's $\delta^{m, \pm 1}$. As a consequence, in the calculation of correlation function $\chi_{x x}$ only the matrices $\hat{\Gamma}_{1}^{m= \pm 1}$ and $\hat{\Gamma}_{2}^{m= \pm 1}$ are involved.

Performing the necessary matrix multiplications and the remaining frequency summation one gets for the dynamical part of the spin correlation function

$$
\begin{aligned}
& \chi_{x x}^{\text {dynamic }}(q=0, \omega) \\
& \quad=\frac{1}{8} \nu\left(\epsilon_{F}\right)\left(1+\Gamma_{2}^{0}\right)^{2} \\
& \quad \times \frac{-\Delta^{2}+2 \omega^{2}\left(1+\Gamma_{2}^{1}\right)\left(1+\Gamma_{2}^{2}\right)}{\Delta^{2}\left[1+\left(\Gamma_{2}^{0}+\Gamma_{2}^{2}\right) / 2\right]-\omega^{2}\left(1+\Gamma_{2}^{0}\right)\left(1+\Gamma_{2}^{1}\right)\left(1+\Gamma_{2}^{2}\right)} .
\end{aligned}
$$

Here we performed an analytic continuation from the positive frequencies on the Matsubara axis to the real frequency axis by $i \omega_{n} \rightarrow \omega$. Together with the static part of $\chi_{x x}^{\text {static }}(\omega=0)=(1 / 4) \nu\left(\epsilon_{F}\right)\left(1+\Gamma_{2}^{0}\right)$ this leads to the final result

$$
\begin{aligned}
& \chi_{x x}^{\text {total }}(q=0, \omega) \\
& \quad=\frac{1}{8} \nu\left(\epsilon_{F}\right)\left(1+\Gamma_{2}^{0}\right) \\
& \quad \times \frac{\Delta^{2}\left(1+\Gamma_{2}^{2}\right)}{\Delta^{2}\left[1+\left(\Gamma_{2}^{0}+\Gamma_{2}^{2}\right) / 2\right]-\omega^{2}\left(1+\Gamma_{2}^{0}\right)\left(1+\Gamma_{2}^{1}\right)\left(1+\Gamma_{2}^{2}\right)}
\end{aligned}
$$

Remarkably, this expression reduces to Eq. (33) obtained phenomenologically

$$
\chi_{x x}(q=0, \omega \rightarrow 0)_{\alpha \neq 0}=\frac{1}{8} \frac{\nu\left(\epsilon_{F}\right)}{1+\frac{1}{2}\left[G^{0}+G^{2}\right]},
$$

when the relation

$$
\left(1+G^{m}\right)=1 /\left(1+\Gamma_{2}^{m}\right)
$$

is applied [see Chap. 2, $\S 18$ in Ref. 25 and notice that in Eqs. (18.7) and (18.9) of the textbook $C=-\Gamma_{2}$ and $\left.B=-\Gamma_{2}+2 \Gamma_{1}\right]$. The resonance frequency obtained in Eqs. (49) and (50) reproduces correctly the frequency of the CSR as given by Eq. (31).

\section{RENORMALIZATIONS OF DISORDER-INDUCED RESONANCE BROADENING AND SPIN-RELAXATION RATE}

In this section we first extend the treatment of the dynamic spin susceptibility to include the disorder. This provides us with a source of the spin relaxation, which leads to the broadening of the chiral spin resonance. Next we consider the coupling of the spin degrees of freedom to the electromagnetic field through the current operator. (For the sake of brevity the electric-dipole interaction as a driving force of the spin resonance was ignored in Sec. III where the coupling via the magnetic moment only was considered.) We show, however, that in excitation of the CSR the electric-dipole interaction is, by far, dominating. Eventually, we compare the intensity of the dissipation through the resonant transitions with the nonresonant ac-Drude losses.

To account for the impurities, the RA sections in the above calculations of the spin susceptibility should be replaced by the diffusion ladders. After the standard averaging over the impurities the effective scattering amplitude due to disorder is equal to $\hat{\Xi}=$ $n_{\mathrm{imp}} u^{2}\left(\theta_{\mathbf{p p}^{\prime}}\right) \delta_{\alpha_{1} \alpha_{2}} \delta_{\alpha_{3} \alpha_{4}}$, where $n_{\mathrm{imp}}$ is the number of impurities per unit square, $u\left(\theta_{\mathbf{p p}^{\prime}}\right)$ is the matrix element of the impurity potential, and the Kronecker's symbols describe the spin structure of $\hat{\Xi}$. It is assumed that for electrons at the Fermi energy $u\left(\theta_{\mathbf{p} \mathbf{p}^{\prime}}\right)$ is a function of the scattering angle only. The disorder averaged Green's functions are

$$
G_{\zeta}^{R A}(i \epsilon, \mathbf{p})=\frac{1}{i \epsilon-\epsilon(\mathbf{p})-\zeta \Delta / 2 \pm i / 2 \tau},
$$

where the scattering rate $1 / \tau=\pi \nu\left(\epsilon_{F}\right) n_{i m p}\left\langle u^{2}\left(\theta_{\mathbf{p p}^{\prime}}\right)\right\rangle_{\theta}$. For weak enough SO interaction the scattering rate $1 / \tau$ is independent of the chirality $\zeta$. Note also that the static amplitude $\hat{\Gamma}^{k}$ can be taken ignoring the influence of the disorder when $1 / \epsilon_{F} \tau \ll 1$ based on the arguments presented in the beginning of Sec. III

To study the spin-density correlation function we sum the ladder diagrams describing the two-particle propagation function in the electron-hole channel. For 
a clean system this propagation function is equal to $\left[\left(G^{R} G^{A}\right)^{-1}-\left(\omega_{n} / 2 \pi\right) \Gamma^{k}\right]^{-1}$. Now the multiple rescattering induced by the impurity amplitude $\hat{\Xi}$ and by the $e$-e interaction amplitude $\hat{\Gamma}^{k}$ should be considered simultaneously. The impurity amplitude $\hat{\Xi}$, unlike $\hat{\Gamma}^{k}$, preserves the frequency of the scattered electrons, and therefore in the two-particles diagrams it is not accompanied by the frequency summation. Hence, to include the impurity scattering $\hat{\Xi}$ in the two-particles propagation function it suffices just to modify the previous result as follows: $\left[\left(G^{R} G^{A}\right)^{-1}-\hat{\Xi}-\left(\omega_{n} / 2 \pi\right) \hat{\Gamma}^{k}\right]^{-1}$. As a result, for the total spin-spin correlation function one obtains [compare with Eq. [50)]

$$
\chi_{x x}^{\text {total }}(q=0, \omega)=\frac{1}{8} \nu\left(\epsilon_{F}\right)\left(1+\Gamma_{2}^{0}\right) \frac{\Delta^{2}\left(x_{2} \omega_{n}+1 / \tau_{2}\right)}{\Delta^{2}\left[\left(x_{0}+x_{2}\right) \omega_{n} / 2+1 / 2 \tau_{2}\right]+x_{0} \omega_{n}\left(x_{1} \omega_{n}+1 / \tau_{1}\right)\left(x_{2} \omega_{n}+1 / \tau_{2}\right)} .
$$

Here the scattering rates $1 / \tau_{1}$ and $1 / \tau_{2}$ are determined by the impurity scattering potential as follows: $1 / \tau_{m}=$ $\pi \nu\left(\epsilon_{F}\right) n_{i m p}\left\langle(1-\exp (-i m \theta)) u^{2}\left(\theta_{\mathbf{p p}^{\prime}}\right)\right\rangle_{\theta}$ with $m=1,2$, while the frequency renormalization factors $x_{0,1,2}=1+$ $\Gamma_{2}^{0,1,2}$. Since it is $\Gamma_{2}$ which controls the interaction in the spin-density channel, the correlation function (54) is determined only by the coefficients of the angular expansion of this amplitude.

Equation (54) reveals the existence of the CSR when the system is clean enough, $\Delta \gg 1 / \tau_{1,2}$. To determine the position and width of the resonance, one has to perform the analytical continuation of the retarded correlation function (54) from the Matsubara axis to the real one and to find the roots of the cubic polynomial in the denominator of Eq. (54). In the vicinity of the resonance, the spin-density correlation function can be written as

$$
\chi_{x x}^{\mathrm{total}}(q=0, \omega)=-\frac{1}{8} \nu\left(\epsilon_{F}\right) \frac{\left(1+\Gamma_{2}^{0}\right)}{\left(1+x_{2} / x_{0}\right)} \frac{\omega_{\mathrm{res}}^{2}}{\omega^{2}-\omega_{\mathrm{res}}^{2}+2 i \eta_{2} \omega} .
$$

The renormalized frequency and the width of the CSR are, correspondingly,

$$
\omega_{\mathrm{res}}=\Delta\left(\frac{1+x_{2} / x_{0}}{2 x_{1} x_{2}}\right)^{1 / 2}
$$

and

$$
\eta_{2}=\frac{1}{2 x_{2}\left(1+x_{2} / x_{0}\right) \tau_{2}}+\frac{1}{2 x_{1} \tau_{1}} .
$$

Under the condition of stability of the electron liquid all the parameters $x_{0,1,2}$ are positive (no Pomeranchuk's instabilities) and $\eta>0$. The positive sign of $\eta_{2}$ corresponds to the attenuation of the spin-density excitations as it should be.

The third pole, which is purely imaginary, $\eta_{1}=$ $-i /\left[\left(x_{0}+x_{2}\right) \tau_{2}\right]$, describes the relaxation rate of the "chiral magnetization". Both relaxation rates $\eta_{1,2}$ are determined by the combinations of the scattering rates $1 / \tau_{1,2}$ only, which is natural for $\Delta \gg 1 / \tau_{1,2}$.

Note that the structure of the denominator in Eq. (54) is rich enough that regimes with other relaxation rates and different parameters of the resonance are possible when $1 /\left(x_{1} \tau_{1}\right), 1 /\left(x_{2} \tau_{2}\right)$, and $1 /\left[\left(x_{0}+x_{2}\right) \tau_{2}\right]$ differ significantly from each other. This is likely to happen near an instability when one of $x_{0,1,2} \gg 1$.

In the limit when the SO interaction is small (i.e., when $\left.\Delta \ll 1 / \tau_{1,2}\right)$, it follows from Eq. (54) that the rate of the spin relaxation is determined by the D'yakonov-Perel mechanism ${ }^{23}$ with a proper Fermi-liquid renormalization

$$
\begin{aligned}
\chi_{x x}^{\text {total }}(q= & 0, \omega)= \\
& \frac{1}{8} \nu\left(\epsilon_{F}\right)\left(1+\Gamma_{2}^{0}\right) \frac{\Delta^{2} \tau_{1}}{\Delta^{2} \tau_{1} / 2+\left(1+\Gamma_{2}^{0}\right) \omega_{n}} .
\end{aligned}
$$

Let us now discuss the mechanisms of excitation of the CSR. The peculiar feature of the SO systems is that the single-particle current operator $\mathbf{J}$ contains spin [see Eq. (11)]. Consequently, the electric-dipole interaction $-(e / c) \mathbf{A} \mathbf{J}$ couples the electromagnetic field $\mathbf{A}$ to the spin density. The electric-dipole interaction is a much more effective way of excitation of the spin resonance compared to coupling of the electromagnetic wave to the magnetic moments via the Zeeman interaction. (To excite the CSR it is necessary to have an in-plane component of the electric field of the radiation. This can be achieved either in the Faraday geometry when the electromagnetic wave is incident along the direction perpendicular to the plane of the 2DEG or in the extraordinary Voigt geometry when the electromagnetic wave propagates parallel to the plane of the 2DEG with the in-plane electric field.) To clarify this issue, consider the electromagnetic wave in the Faraday geometry with $\mathbf{E}(t)=\hat{x} E_{0} e^{i k z-i \omega t}$ and $\mathbf{B}(t)=\hat{y} E_{0} e^{i k z-i \omega t}$. The energy dissipation rate according to the Kubo formalism is determined by

$$
Q=2 \operatorname{Re} \sigma_{x x}(\omega) E_{0}^{2}+2 \omega\left(g \mu_{B}\right)^{2} \operatorname{Im} \chi_{y y}(\omega) E_{0}^{2}
$$

(Note that at the absence of the external magnetic field there is no superposition contribution from the electric and magnetic-dipole interactions to $Q$.) For the purpose of comparison of the two mechanisms, let us confine $\sigma_{x x}(\omega)$ to the contribution originating from spin transitions. When only the spin-term of the current operator is kept in the correlation function determining the 
conductivity, it follows immediately that $\operatorname{Re} \sigma_{x x}(\omega)=$ $\left(4 e^{2} \alpha^{2} / \omega\right) \operatorname{Im} \chi_{y y}(\omega)$ and the dissipation rate $Q$ can be written as

$$
Q=2 \omega \operatorname{Im} \chi_{y y}(\omega)\left[\frac{4 e^{2} \alpha^{2}}{\omega^{2}}+\left(g \mu_{B}\right)^{2}\right] E_{0}^{2} .
$$

For $\omega=\omega_{\text {res }}$ the first term in the square brackets does not depend on the SO-coupling constant $\alpha$. Omitting all renormalizations, it is $\sim e^{2} \hbar^{2} / p_{F}^{2}$, while the second term can be estimated as $\sim e^{2} \lambda^{2}$, where the Compton's length $\lambda=\hbar / m_{e} c$. The dipole moment corresponding to the wavelength of the electrons is few orders of magnitude larger than the dipole moment corresponding to the Compton's length, and therefore only the electricdipole mechanism is relevant for the excitation of the CSR. The relative strength of the two mechanisms is $(\lambda / \lambda)^{2} \sim 10^{-9}-10^{-8}$.

Ignoring the momentum part of the current operator in the correlation function that determines $\sigma_{x x}(\omega)$ is justified in the clean system only, i.e., when the total momentum is conserved. In the presence of disorder the situation is more subtle. Namely, in the limit $\omega \ll 1 / \tau$ the momentum part of the current operator participates equally in the excitation of the spin-flip transitions. Moreover, there are claims that in the static limit the spin-flip transitions cannot be excited through the electric-dipole interaction, $-(e / c) \mathbf{A J}$, because there is a complete cancellation between the two terms of the current operator. (This cancellation has been noticed in Refs, 12,13,14,15,16 in the context of vanishing of the static spin-Hall conductivity in the bulk of a macroscopic system.) In the high-frequency limit the balance between different terms of the current operator is changed, and participation of the momentum part of the current operator in the excitation of the spin-flip transitions becomes insignificant.

Let us clarify the action of the different terms of the current on the spin-flip transitions when the frequency is finite. Suppose that the current-current correlation function begins with the momentum part of the current operator. Naively it cannot excite the spin transitions because $\operatorname{Tr} \int d \xi \mathbf{p}_{F} \hat{\mathcal{G}}^{R}(i \epsilon, \mathbf{p}) \sigma_{x} \hat{\mathcal{G}}^{A}(i \epsilon, \mathbf{p}) \propto \int d \xi f_{\text {odd }}(\xi)$, where $f_{\text {odd }}(\xi)$ is an odd function of $\xi$. To get a nonvanishing contribution to the spin transitions from these terms one has to keep the dependence on $\xi$ either in the current vertex or in the spin-splitting of the energy spectrum (both depend explicitly on the momentum). This will inevitably be accompanied by the appearance of the small parameter $\alpha / v_{F}$. However, the spin part of the current contains the same parameter because it also originates from the SO interaction. Together the two terms in the current operator give a frequency-dependent factor $g_{A \rightarrow S}=\left(\alpha / v_{F}\right)\left[1-1 /\left(\omega_{n} \tau+1\right)\right]$ for the effective coupling of the electromagnetic field $\mathbf{A}$ to spins (here, for the sake of brevity, impurities are assumed to be pointlike). At low frequencies this factor approaches zero making the excitation of the spin-flip transitions problematic. At finite frequencies the second term in $g_{A \rightarrow S}$ originating from the momentum part of the current operator decreases, resulting in a finite $g_{A \rightarrow S}$. This is why in the Introduction we have pointed out that it is worthwhile to turn to AC phenomena for studying the effects of the $\mathrm{SO}$ interaction. Note that the CSR is a high-frequency phenomenon. For the CSR to be narrow enough the resonance frequency $\omega_{\text {res }}$ should much exceed $\eta_{2} \sim 1 / \tau_{1,2}$. In this limit the factor $\left[1-1 /\left(\omega_{n} \tau+1\right)\right]$ in $g_{A \rightarrow S}$ approaches 1 .

For completeness let us discuss the spin-Hall conductivity in the static limit. As it has been pointed out in Ref. 13, the statement that the spin-Hall conductivity vanishes is valid only inside the bulk of a macroscopic system. Namely, the cancellation between the two terms of the current operator has been demonstrated for $\varsigma_{x y}^{z}(q=0 ; \omega \ll 1 / \tau)$, i.e., in a system of infinite size. Still, in a finite-size system the spin-Hall phenomenon can exist as the vanishing of the factor $g_{A \rightarrow S}$ may not work near the edges. In the latter case, in a broad macroscopic system only a small fraction of the longitudinal current that flows within a narrow strip near the edges is effective for the spin-Hall voltage as the spin-Hall conductivity degrades inside the bulk of the sample. For disconnected (or weakly tunneling) edges the existence of a non-zero spin conductivity results in the accumulation of a $z$-component of spin density at the edges. In this connection, let us indicate that the spin-Hall effect reported in Ref. 17 has been observed just at the edges of the conducting channel.

\section{CONCLUDING DISCUSSION}

The analysis of the equations of motion performed along the lines of the argumentation of the Kohn's theorem reveals an inherent relationship between a transverse conductivity and a corresponding resonance in a clean system. The same correlation function that describes the resonance determines the value of the transverse conductivity, including its static limit. Such relationship is useful for understanding the properties of the transverse conductivity. For example, in Sec. II] we demonstrate that the absence of the $e-e$ renormalizations to the Hall coefficient in a clean system is a direct consequence of the Kohn's theorem for the frequency of the cyclotron resonance. With this in mind, in Sec. III we find the connection between the spin-Hall conductivity and a spin-resonance in a 2DEG with the SO interaction. Since this spin resonance occurs as a result of the transitions between the electron states of different chirality which are split by the SO interaction, it is called in this paper a chiral spin resonance.

Recently, considerable efforts have been made to determine the value of the SO splitting in semiconductor heterostructures from the measurements of the magnetoresistance 29.30 .31 Another standard method for measuring the SO splitting in the electron energy spectrum in 2DEG is the analysis of the positions of the nodes 
in the beating pattern of the Shubnikov-de Haas $(\mathrm{SdH})$ oscillations 32.33 .34 .35 .36 This method, however, has certain reservations ${ }^{29}$ In particular, the $\mathrm{SdH}$ oscillations are controlled by the single-particle relaxation time $\tau$, which in heterostructures is significantly shorter than the transport time. In Sec. $\nabla$ we show that the width of the CSR can be much smaller than $1 / \tau$ as it is controlled by the scattering rates $1 / \tau_{1,2}$. The CSR, if observed, can be a useful tool for an accurate measurement of the strength of the SO interaction.

Let us discuss the questions of the excitation and detection of the CSR. The CSR is a limiting case of the combined resonance ${ }^{18}$ when a static magnetic field is absent. The peculiar feature of the combined ESR (including the CSR) is that it can be excited by the electricdipole interaction $-(e / c) \mathbf{A J}$ rather than by coupling of the electromagnetic wave to the magnetic moments via the Zeeman interaction. ${ }^{20}$ The possibility of the electricdipole excitation of the resonance makes the observation the combined ESR a feasible task even in 2D systems.

A problematic point in detecting of a spin resonance in $2 \mathrm{D}$ systems is that a number of electrons available for spin transitions is small. A standard method to overcome this difficulty is detecting the ESR by the microwaveinduced change of the magnetoresistivity. The resonance frequency measured in this way ${ }^{37.38}$ when extrapolated to zero magnetic field indicates the existence of an intrinsic spin splitting. Bychkov and Rashba 5 attributed this splitting to the SO interaction induced by the structure inversion asymmetry and extracted the value of the SO coupling constant $\alpha$.

To observe a resonance a fine-tuning control over the resonance frequency is needed. An external magnetic field used commonly in ESR experiments may not be welcome for this purpose. The in-plane magnetic field makes the spin splitting anisotropic along the Fermi surface, whereas the perpendicular magnetic field requires an interpolation of the resonance frequency to a zerofield limit. In addition, the orbital quantization induced by the perpendicular magnetic field rapidly leads to the quantization of the energy levels resulting in the quantum Hall-effect regime as it took place in Refs. 37 and 38. Perhaps, for the CSR it is preferable to avoid the use of the magnetic field and instead to analyze the resonance by combining the transport measurements with the spectroscopy analysis. ${ }^{39}$ Another possible solution of the tuning problem in the case of the CSR is the gatevoltage control of the SO splitting. For GaAs it does not look very promising as the shift of the resonance frequency is rather small ${ }^{40}$ However, it is known that in $\operatorname{In}_{x} \mathrm{Ga}_{1-x}$ As the gate voltage strongly affect the spin splitting that allows the resonance frequency to vary in a broad range 34.35 .36

It is useful to compare the energy absorption related to the resonant spin-flip transitions with the nonresonant heating of the 2DEG (Drude mechanism). Assuming that the microwave radiation has a narrow frequency range compared to the width of the resonance, one can estimate

\begin{tabular}{lccccc}
\hline \hline & & $\epsilon_{F}$ & $\Delta$ & $\hbar / \tau_{\text {tr }}$ & \\
& $n \times 10^{11} \mathrm{~cm}^{-2}$ & $(\mathrm{meV})$ & $(\mathrm{meV})$ & $(\mathrm{meV})$ & $\Upsilon$ \\
\hline InGaAs & 20 & 100 & 5 & 0.1 & 5 \\
GaAs & 2 & 7 & 0.07 & 0.0008 & 1 \\
\hline \hline
\end{tabular}

TABLE I: Electronic properties of 2DEG with SO interaction.

the resonant part of the losses as $\sim e^{2} \nu \alpha^{2} \tau_{t r} E_{0}^{2} \sim e^{2} \Upsilon E_{0}^{2}$. At $\omega \approx \omega_{\text {res }} \sim \Delta$, the Drude part of the dissipation is $\sim e^{2} \nu v_{F}^{2} \tau_{t r} /\left(\Delta \tau_{t r}\right)^{2} E_{0}^{2} \sim e^{2} \Upsilon^{-1} E_{0}^{2}$. The dimensionless parameter $\Upsilon=\left(\Delta \tau_{t r}\right)\left(\Delta / \epsilon_{F}\right)$ is a product of two competing factors. The factor $\Delta / \epsilon_{F}$ characterizes the relative strength of the SO interaction, and it is relatively small, whereas the quality factor $\Delta \tau_{t r} \gg 1$. The ratio of the two contributions to the energy absorption is $\sim \Upsilon^{2}$.

The characteristics of the 2DEG and the data about the SO splitting are presented in Table \. There, we assume that the mobility $\mu_{e}$ of $\operatorname{In}_{x} \mathrm{Ga}_{1-x} \mathrm{As}$ is about $2 \times 10^{5} \mathrm{~cm}^{2} / \mathrm{Vs}$, which is available for the present samples. For GaAs we take $\mu_{e}=20 \times 10^{6} \mathrm{~cm}^{2} / \mathrm{Vs}$ available only for the best reported samples. The value of the SO splitting for $\operatorname{In}_{x} \mathrm{Ga}_{1-x} \mathrm{As}$ is taken from Ref. 34, where it was extracted from the beat pattern of SdH oscillations. For GaAs the experimental scale of the SO interaction, $\sim 100 \mathrm{~ns}^{-1}$, is taken from Fig. 3 of Ref. 30. We see that the resonance frequency in $\operatorname{In}_{x} \mathrm{Ga}_{1-x}$ As corresponds to the far-infrared range, whereas in GaAs the relevant frequencies are in the millimeter wave range. To observe the CSR the spin splitting induced by the SO interaction should be sufficiently isotropic. This may be realized in various situations (see Appendix[B for more details). One example is the asymmetrical quantum well where the SO interaction of the structure inversion asymmetry origin is dominant. Another variant is to fabricate a symmetric quantum well with the [001]-growth direction and, in this way, get rid of the SIA spin-orbit interaction leaving only the SO interaction because of the lack of inversion symmetry of the host crystal (BIA) $2^{2}$ The last example is [111]-grown quantum well, which can be asymmetrical, where the combined action of the SO interactions SIA and BIA results in the isotropic spin splitting. It is generally accepted that in $\operatorname{In}_{x} \mathrm{Ga}_{1-x}$ As heterostructures the dominant $\mathrm{SO}$ interaction is because of the SIA 34 However, this may be not the case for the GaAs heterostructures where the BIA spin-orbit interaction is of comparable strength to the SO interaction induced by the interface electric field. The resulting spin splitting is anisotropic on the Fermi surface. This makes an observation of the CSR in a [001]-grown GaAs heterostructure problematic.

In view of the considerable progress in the quality of $2 \mathrm{D}$ heterostructures it is worthwhile to extend the measurements of ESR to zero magnetic field. This can give a direct information about the strength of the SO interaction. 


\section{APPENDIX A: CALCULATION OF SPIN-HALL CONDUCTIVITY IN THE ABSENCE OF $e-e$ INTERACTION}

Let us calculate the spin-Hall conductivity $\varsigma_{x y}^{z}$ for noninteracting particles, copying the logic of the calculation of $\sigma_{x y}$ in Sec. II] [Equations A1 -A3 duplicate the corresponding equations in Secs. III and III We repeat them here to make this Appendix self-contained.] A single-particle Hamiltonian with the Bychkov-Rashba $\mathrm{SO}$ interaction ${ }^{5}$ is

$$
H_{i}^{S O}=\frac{\boldsymbol{p}_{i}^{2}}{2 m}+\alpha\left[\boldsymbol{p}_{i} \times \boldsymbol{\ell}\right] \cdot \boldsymbol{\sigma}_{i},
$$

where the unit vector $\ell$ is perpendicular to the plane of the $2 \mathrm{DEG}$. As a result, the energy spectrum is split into two chiral branches $\epsilon_{p}^{ \pm}=p^{2} / 2 m \pm \alpha p$.

In the presence of the $\mathrm{SO}$ interaction the single-particle current operator $\boldsymbol{j}_{i}$ contains a spin-dependent term

$$
\boldsymbol{j}_{i}=\frac{\boldsymbol{p}_{i}}{m}+\alpha\left[\boldsymbol{\ell} \times \boldsymbol{\sigma}_{i}\right]
$$

Since in the absence of a magnetic field the Hamiltonian (A1) does not contain any coordinate dependence, the momentum part of the current is time independent. Still, the current has dynamics as the current operator contains spin.

We analyze the dynamics of spin in the chiral basis with the rotated Pauli matrices $\tau_{\mathbf{p}}^{\nu}=\left(\mathbf{a}_{\mathbf{p}}^{\nu} \cdot \boldsymbol{\sigma}\right)$, where $\mathbf{a}_{\mathbf{p}}^{\nu}=\left\{\mathbf{a}^{1}, \mathbf{a}^{2}, \mathbf{a}^{3}\right\}=\{-\boldsymbol{\ell}, \hat{\mathbf{p}}, \hat{\mathbf{p}} \times \boldsymbol{\ell}\}$ and $\hat{\mathbf{p}}$ stands for a unit vector in the direction of momentum $\mathbf{p}$. In the chiral basis the free Hamiltonian (A1) acquires the diagonal form

$$
H_{i}^{S O}=\frac{\mathbf{p}_{i}^{2}}{2 m}+\alpha\left|\mathbf{p}_{i}\right| \tau_{\mathbf{p}_{i}}^{3}
$$

with the diagonal elements equal to $\epsilon_{p}^{ \pm}$.

Any operator of the form $T_{i}^{ \pm}=f\left(\left|\mathbf{p}_{i}\right|\right) \tau_{\mathbf{p}_{i}}^{ \pm}$has an equation of motion

$$
\frac{d}{d t} T_{i}^{ \pm}=i\left[H_{i}^{S O}, T_{i}^{ \pm}\right]= \pm i \omega_{p_{i}}^{s o} T_{i}^{ \pm}
$$

where $\tau^{ \pm}$are defined in the usual way, $\tau^{ \pm}=\left(\tau^{1} \pm i \tau^{2}\right) / 2$, and

$$
\omega_{p}^{s o}=\epsilon_{p}^{+}-\epsilon_{p}^{-}=2 \alpha p .
$$

These equations allows us to find the time dependence of the current operators $j_{i}^{x}$ and $j_{i}^{y}$. For that, we express the current components in terms of the $\tau$-matrices

$$
\begin{aligned}
j^{x} & =\frac{p^{x}}{m}+\alpha \frac{p^{x}}{p} \tau_{\mathbf{p}}^{3}-\alpha \frac{p^{y}}{p} \tau_{\mathbf{p}}^{2}, \\
j^{y} & =\frac{p^{y}}{m}+\alpha \frac{p^{y}}{p} \tau_{\mathbf{p}}^{3}+\alpha \frac{p^{x}}{p} \tau_{\mathbf{p}}^{2},
\end{aligned}
$$

where the particle's index $i$ is omitted. The first two terms in the expressions $j^{x, y}$ do not lead to the transitions between the chiral states of $H_{i}^{S O}$ and therefore do not depend on time. The terms with $\tau^{2}$ matrix induce the transitions between the states of the opposite chirality separated by the energy $\pm \omega^{\text {so }}$. In result, the time dependence of the current component $j^{y}(t)$ is

$$
j^{y}(t)=\frac{p^{y}}{m}+\frac{\alpha}{p}\left[p^{y} \tau_{\mathbf{p}}^{3}+p^{x}\left(\tau_{\mathbf{p}}^{2} \cos \omega_{p}^{s o} t+\tau^{1} \sin \omega_{p}^{s o} t\right)\right],
$$

and a similar expression for $j^{x}(t)$.

The oscillatory terms in Eq. (A8) are analogous to the oscillations in the cyclotron resonance that originate from the transitions between the states with different circulation; compare Eqs. (5) and (A4). Furthermore, the same description applies for the case of the ESR, where the transitions in the external magnetic field occur between the states of the opposite spin polarization.

We are currently in the stage when the application of the Kohn's argumentation for the time evolution of the current operators allows us to calculate the transverse conductivity. The transverse spin conductivity $\varsigma_{x y}^{z}$ describes the response of the spin-z-component current in the $x$ direction, $\mathfrak{j}_{z}^{x}=\frac{1}{4}\left(\sigma^{z} j^{x}+j^{x} \sigma^{z}\right)$, to the electric field applied in the $y$ direction. In the chiral basis $\sigma^{z}=-\tau^{1}$, and therefore $\mathfrak{j}_{z}^{x}=-\left(p^{x} / 2 m\right) \tau^{1}$. The transverse spin conductivity is given by the corresponding Kubo formula (we restore the particles index $i$ )

$$
\varsigma_{x y}^{z}=\frac{e}{\omega} \int_{0}^{\infty} d t e^{i \omega t}\left\langle\sum\left[\mathfrak{j}_{z ; i}^{x}, j_{i}^{y}(-t)\right]\right\rangle,
$$

which with the use of Eq. (A8) yields

$$
\varsigma_{x y}^{z}=e \sum\left\langle\frac{\alpha}{m p_{i}}\left[-\frac{p_{i}^{x} p_{i}^{y} \tau_{\mathbf{p}_{i}}^{2}}{\omega^{2}}+\frac{\left(p_{i}^{x}\right)^{2} \tau_{\mathbf{p}_{i}}^{3}}{\omega^{2}-\left(\omega_{p_{i}}^{s o}\right)^{2}}\right]\right\rangle .
$$

To get the final result one has to perform the average $\langle\cdots\rangle$ in this equation. When averaged, the term with $\tau^{2}$ matrix vanishes, $\left\langle\tau_{\mathbf{p}}^{2}\right\rangle=0$, because the spindependent term in Hamiltonian A1 contains $\tau^{3}$ matrix only. As the chiral states are eigenstates with energies $\epsilon_{\mathbf{p}}^{ \pm}$, the population of a state $\mathbf{p}$ is equal to $n_{F}\left(\epsilon_{\mathbf{p}}^{+}\right)$for the + chirality state and $n_{F}\left(\epsilon_{\mathbf{p}}^{-}\right)$for the - chirality state; $n_{F}(\epsilon)=[\exp (\epsilon-\mu)+1]^{-1}$. Correspondingly, the expected value of $\tau_{\mathbf{p}}^{3}$ is equal to $\left\langle\tau_{\mathbf{p}}^{3}\right\rangle=n_{F}\left(\epsilon_{p}^{+}\right)-n_{F}\left(\epsilon_{p}^{-}\right)$. Finally, this yields

$$
\begin{aligned}
\varsigma_{x y}^{z} & =e \int \frac{d^{2} p}{(2 \pi)^{2}} \frac{1}{8 \alpha m p}\left[n_{F}\left(\epsilon_{p}^{-}\right)-n_{F}\left(\epsilon_{p}^{+}\right)\right] \\
& =\frac{e}{8 \alpha m} \int \frac{d p}{2 \pi}\left[n_{F}\left(\epsilon_{p}^{-}\right)-n_{F}\left(\epsilon_{p}^{+}\right)\right] .
\end{aligned}
$$

Unlike, the cyclotron resonance, where all electrons precess together and contribute equally to $\sigma_{x y}=$ $-\left(n e^{2} / m\right) \omega_{c}^{-1}$, in the case of spin-Hall conductivity the contribution from electrons of the opposite chirality tend to cancel each other out. The factor $1 / \alpha$ in Eq. A11 is equivalent to $1 / \omega_{c}$ in $\sigma_{x y}$, but due to the cancellation only a stripe of the width $2 \alpha m$ between the Fermi 
surfaces of electrons of the opposite chirality contributes that makes the value $\varsigma_{x y}^{z}$ finite in the limit of small $\alpha$. For non-interacting electrons it is also possible to express $\varsigma_{x y}^{z}$ as a contribution from the bottom of the band. For that, rewrite $\epsilon_{p}^{ \pm}$as $\epsilon_{p}^{ \pm}=\frac{1}{2 m}(p \pm \alpha m)^{2}-\frac{1}{2} m \alpha^{2}$ and shift the momentum variables to $q^{ \pm}=p \pm \alpha m$. Then

$$
\varsigma_{x y}^{z}=\frac{e}{4 \alpha m} \int_{0}^{\alpha m} \frac{d q}{2 \pi} n_{F}\left(\frac{q^{2}}{2 m}-\frac{m \alpha^{2}}{2}\right) .
$$

At low temperatures $n_{F}(0)=1$ and $\varsigma_{x y}^{z}=e / 8 \pi$ for any finite $\alpha$. On the other hand, when $\alpha m \ll p_{F}$ one can get for arbitrary temperatures $\varsigma_{x y}^{z} \approx(e / 8 \pi) n_{F}(\epsilon=0 ; T)$, where $n_{F}(\epsilon=0 ; T)$ is the occupation number at the bottom of the band at a temperature $T$.

The expression for $\varsigma_{x y}^{z}$ as given by Eq. A11 reminds the corresponding expression for the static spin susceptibility: $\chi=$ $\left(g \mu_{B} / 2 B\right) \int\left[d^{2} p /(2 \pi)^{2}\right]\left[n_{F}\left(\epsilon_{p}-\frac{1}{2} \Delta_{Z}\right)-n_{F}\left(\epsilon_{p}+\frac{1}{2} \Delta_{Z}\right)\right]$, where $\Delta_{Z}=g \mu_{B} B$. This gives a hint why there exists a connection ${ }^{22.41}$ between the spin-density correlation function and $\varsigma_{x y}^{z}$. In Section [I a direct connection between $\varsigma_{x y}^{z}$ and the dynamic (retarded) spin susceptibility has been derived [see Eqs. (14)-(16)]

$$
\begin{aligned}
\varsigma_{x y}^{z} & =\frac{e}{m} \chi_{x x}(q=0, \omega), \\
\chi_{x x}(q & =0, \omega)=\frac{i}{4} \int_{0}^{\infty} d t e^{i \omega t}\left\langle\sum\left[\sigma_{i}^{x}(t), \sigma_{i}^{x}(0)\right]\right\rangle .
\end{aligned}
$$

A direct calculation of $\chi_{x x}(q=0, \omega)$ in the presence of the SO interaction can be done straightforwardly with the help of Eq. A8 . In the limit of small frequencies, $\omega \ll \omega^{s o}$, the correlation function $\chi_{x x}(q=0, \omega \rightarrow 0)_{\alpha \neq 0}$ is equal to a half of the static spin susceptibility of a free 2DEG in the absence of the SO interaction

$$
\chi_{x x}(q=0, \omega \rightarrow 0)_{\alpha \neq 0}=\frac{m}{8 \pi} .
$$

Correspondingly, in the presence of the SO interaction the spin-Hall conductivity $\varsigma_{x y}^{z}=e / 8 \pi$.

\section{APPENDIX B: FERMI-LIQUID ANALYSIS OF SPIN RESONANCE IN THE PRESENCE OF DRESSELHAUS SO INTERACTION (BIA)}

In this appendix we analyze the kinetics of electrons in the presence of the $\mathrm{SO}$ interaction of the BIA origin ${ }^{2}$ The spin-orbit interaction in the semiconductors with the zinc-blende crystal structure is described by the Hamiltonian

$$
H_{\mathrm{bulk}}^{\mathrm{SO}}=\gamma\left[\sigma_{x} k_{x}\left(k_{y}^{2}-k_{z}^{2}\right)+\text { c.p. }\right],
$$

where c.p. stands for the cyclic permutations. For 2DEG the Hamiltonian (B1) leads to a linear in momentum term in the SO interaction $\stackrel{42}{=}$ For the case of the [001]grown quantum well the linear term can be obtained from
$H_{\text {bulk }}^{\mathrm{SO}}$ by replacing $k_{z}^{2}$ and $k_{z}$ by their averages $\left\langle k_{z}^{2}\right\rangle$ and $\left\langle k_{z}\right\rangle=0$

$$
H_{[001]}^{\mathrm{SO}}=\beta\left(p_{x} \sigma_{x}-p_{y} \sigma_{y}\right),
$$

where $\beta=-\gamma\left\langle k_{z}^{2}\right\rangle$. Unlike the Rashba Hamiltonian (10), this term does not have a structure of a triple scalar product and, therefore, it is not rotationally invariant. Nevertheless, it leads to the isotropic spin splitting of the energy spectrum.

The structure of the linear term that is formally identical to the Hamiltonian (10) can be realized in the [111]grown quantum well. This is a consequence of a threefold rotation symmetry with respect to [111] axis. On the contrary, SO interaction in the [110]-grown quantum well results in the anisotropic spin splitting and therefore such heterostructures are not suitable for the observation of CSR.

In the following we analyze the kinetic equation (24) for the case [001]-grown 2DEG when the linear SO interaction term is given by Eq. (B2). The remaining cubic terms in the SO interaction is a source of the CSR broadening. It can be neglected for the narrow enough quantum well when $\left\langle k_{z}^{2}\right\rangle \gg k_{F}^{2}$.

It is instructive to start with the Rashba and Dresselhaus interactions acting together. The combined action of the BIA and SIA mechanisms of the spin-orbit interaction is described by the Hamiltonian

$$
\begin{aligned}
H_{\text {linear }}^{\text {SO }} & =\alpha\left(p_{y} \sigma_{x}-p_{x} \sigma_{y}\right)+\beta\left(p_{x} \sigma_{x}-p_{y} \sigma_{y}\right) \\
& =\frac{1}{2} \Delta_{\mathbf{p}}\left[a_{\mathbf{p}} \sigma_{x}+b_{\mathbf{p}} \sigma_{y}\right]
\end{aligned}
$$

where $a_{\mathbf{p}}^{2}+b_{\mathbf{p}}^{2}=1$. In the presence of the two SO interaction terms the spin splitting energy $\Delta_{\mathbf{p}}$ is a varying function along the Fermi surface

$$
\Delta_{\mathbf{p}}=2 p_{F} \Lambda_{\mathbf{p}}, \quad \Lambda_{\mathbf{p}}=\left[\alpha^{2}+\beta^{2}+2 \alpha \beta \sin 2 \theta_{\mathbf{p}}\right]^{1 / 2} .
$$

The coefficients $a_{\mathbf{p}}$ and $b_{\mathbf{p}}$ are defined as

$$
\begin{aligned}
& a_{\mathbf{p}}=\Lambda_{\mathbf{p}}^{-1}\left(\alpha \sin \theta_{\mathbf{p}}+\beta \cos \theta_{\mathbf{p}}\right) \\
& b_{\mathbf{p}}=\Lambda_{\mathbf{p}}^{-1}\left(-\alpha \cos \theta_{\mathbf{p}}-\beta \sin \theta_{\mathbf{p}}\right) .
\end{aligned}
$$

We introduce a set of the Pauli matrices such that the SO spin splitting term takes the form $\delta \epsilon^{S O}=\frac{1}{2} \Delta_{\mathbf{p}} t_{\mathbf{p}}^{3}$, namely,

$$
\begin{aligned}
t_{\mathbf{p}}^{1} & =-\sigma_{z}, \quad t_{\mathbf{p}}^{2}=-b_{\mathbf{p}} \sigma_{x}+a_{\mathbf{p}} \sigma_{y}, \\
t_{\mathbf{p}}^{3} & =a_{\mathbf{p}} \sigma_{x}+b_{\mathbf{p}} \sigma_{y} .
\end{aligned}
$$

The renormalization of the $\beta$ term by the $e$-e interaction is analyzed in the same way as the renormalization of the Bychkov-Rashba coefficient $\alpha$ in Eq. (20) yielding $\beta^{*} / \beta=\alpha^{*} / \alpha=1 /\left(1+G^{1}\right)$.

The kinetic equation similar to Eqs. (27a)-(27c) can now be written using the expansion of $\hat{u}\left(\theta_{\mathbf{p}}\right)$ in terms of 
the $t_{\mathbf{p}}$-matrices: $\hat{u}\left(\theta_{\mathbf{p}}\right)=\sum u_{i}\left(\theta_{\mathbf{p}}\right) t_{\mathbf{p}}^{i}$. The force term is equal to

$\left[\delta \hat{\epsilon}_{\mathbf{p}}^{S O}, \sigma_{x}\right] \mathfrak{F} e^{i \omega t}=-i t^{1}\left(2 p_{F} \alpha^{*} \cos \theta_{\mathbf{p}}+2 p_{F} \beta^{*} \sin \theta_{\mathbf{p}}\right) \mathfrak{F} e^{i \omega t}$

and the kinetic equation acquires the form

$$
\begin{gathered}
\Delta_{\mathbf{p}}^{-1} \frac{d u_{1}\left(\theta_{\mathbf{p}}\right)}{d t}=u_{2}\left(\theta_{\mathbf{p}}\right)+\int d \theta_{\mathbf{p}^{\prime}} G\left(\theta_{\mathbf{p p}^{\prime}}\right) C^{3,2}\left(\theta_{\mathbf{p}}, \theta_{\mathbf{p}^{\prime}}\right) u_{2}\left(\theta_{\mathbf{p}^{\prime}}\right) \\
-\Delta_{\mathbf{p}}^{-1}\left[2 p_{F} \alpha^{*} \cos \theta_{\mathbf{p}}+2 p_{F} \beta^{*} \sin \theta_{\mathbf{p}}\right] \mathfrak{F} e^{i \omega t}, \quad(\mathrm{~B} 8 \mathrm{a}) \\
\Delta_{\mathbf{p}}^{-1} \frac{d u_{2}\left(\theta_{\mathbf{p}}\right)}{d t}=-u_{1}\left(\theta_{\mathbf{p}}\right)-\int d \theta_{\mathbf{p}^{\prime}} G\left(\theta_{\mathbf{p} \mathbf{p}^{\prime}}\right) u_{1}\left(\theta_{\mathbf{p}^{\prime}}\right) .
\end{gathered}
$$

Here the structure factor $C^{3,2}$ appears because of the commutator $\left[t_{\mathbf{p}}^{3}, t_{\mathbf{p}^{\prime}}^{2}\right]=-2 i t^{1} C^{3,2}\left(\theta_{\mathbf{p}}, \theta_{\mathbf{p}^{\prime}}\right)$, where

$$
\begin{aligned}
& C^{3,2}\left(\theta_{\mathbf{p}}, \theta_{\mathbf{p}^{\prime}}\right)=a_{\mathbf{p}} a_{\mathbf{p}^{\prime}}+b_{\mathbf{p}} b_{\mathbf{p}^{\prime}} \\
& \quad=\left(\Lambda_{\mathbf{p}} \Lambda_{\mathbf{p}^{\prime}}\right)^{-1}\left[\left(\alpha^{2}+\beta^{2}\right) \cos \left(\theta_{\mathbf{p}}-\theta_{\mathbf{p}^{\prime}}\right)+2 \alpha \beta \sin \left(\theta_{\mathbf{p}}+\theta_{\mathbf{p}^{\prime}}\right)\right]
\end{aligned}
$$

The structure factor $C^{3,2}$ reduce to $\cos \left(\theta_{\mathbf{p}}-\theta_{\mathbf{p}^{\prime}}\right)$ when only one of the SO interactions (BIA or SIA) is acting.
The kinetic equation has the same form when either the SIA or BIA mechanism acts solely. Hence the pure BIA system exhibits the same chiral spin resonance with , the frequency given by Eq. (31) and $\Delta \rightarrow \Delta_{\text {BIA }}$. Actually this observation, as well as equal renormalization of $\alpha$ and $\beta$, is related to a duality of the $\alpha$ - and $\beta$-SO terms. Namely, $H_{\text {linear }}^{S O}$ is symmetric with respect to a simultaneous rotation of the Pauli matrices around the direction $\hat{n}=(\hat{x}+\hat{y}) / \sqrt{2}$ by $\pi$, i.e., $\sigma_{x} \rightleftarrows \sigma_{y}, \sigma_{z} \rightarrow-\sigma_{z}$, together with the replacement $\alpha \rightleftarrows-\beta$, whereas the $e-e$ interaction is symmetric with respect to any spin rotations.

\section{ACKNOWLEDGMENTS}

We thank Alex Punnoose, Emmanuel Rashba, and Yehoshua Levinson for stimulating discussions of the problems related to the spin-Hall conductivity. We thank Jurgen Smet and Gerhard Abstreiter for a useful discussion of the microwave experiments. The work was supported by the US-Israel Binational Science Foundation (BSF) and the Minerva Foundation.
1 S. Datta and B. Das, Appl. Phys. Lett. 56 , 665 (1990).

2 G. F. Dresselhaus, Phys. Rev. 100, 580 (1955).

3 E.I. Rashba and V. I. Sheka, Fiz. Tverd. Tela (S.Peterburg) 2, 162 (1959).

4 F. T. Vas'ko, Pis'ma Zh. Eksp. Teor. Fiz. 30, 574 (1979) [Sov. Phys.-JETP Lett. 30, 540 (1979)].

${ }^{5} \mathrm{Yu}$. A. Bychkov and E. I. Rashba, Pis'ma Zh. Eksp. Teor. Fiz. 39, 66 (1984) [Sov. Phys.-JETP Lett. 39, 78 (1984)].

6 J. E. Hirsch, Phys. Rev. Lett. 83, 1834 (1999).

7 S. Murakami, N. Nagaosa, and S.-C. Zhang, Science 301, 1348 (2003); S. Murakami, Phys. Rev. B 69, 241202(R) (2004).

8 J. Sinova, D. Culcer, Q. Niu, N. A. Sinitsyn, T. Jungwirth, and A. H. MacDonald, Phys. Rev. Lett. 92, 126603 (2004).

9 J. Hu, B. A. Bernevig, and C. Wu, cond-mat/0310093 (unpublished).

10 J. Schliemann and D. Loss, Phys. Rev. B 69, 165315 (2004).

11 E. I. Rashba, Phys. Rev. B 68, 241315(R) (2003).

12 J.-I. Inoue, G. E. W. Bauer, and L. W. Molenkamp, Phys. Rev. B 70, 041303(R) (2004).

13 E. G. Mishchenko, A. V. Shytov, and B. I. Halperin, Phys. Rev. Lett 93, 226602 (2004).

14 A. Khaetskii, cond-mat/0408136 (unpublished).

15 R. Raimondi and P. Schwab, Phys. Rev. B 71, 033311 (2005).

16 E. I. Rashba, Phys. Rev. B 70, 161201 (2004).

17 We believe that the finite size effects are responsible for the observation of the spin-Hall effect reported in the recent papers by Y. K. Kato, R. C. Myers, A. C. Gossard, and D. D. Awschalom, Science 306, 1910 (2004); and J. Wunderlich, B. Kastner, J. Sinova, and T. Jungwirth,
Phys. Rev. Lett. 94, 047204 (2005). For further discussion see the last paragraph of Sec. D

18 E. I. Rashba Fiz. Tverd. Tela (Leningrad) 2, 1224 (1960) [Sov. Phys.-Solid State 2, 1109 (1960)].

19 B. D. McCombe, Phys. Rev. 181, 1206 (1969).

20 E. I. Rashba and V. I. Sheka, in Landau Level Spectroscopy, edited by G. Landwehr, and E. I. Rashba (North-Holland, Amsterdam, 1991), p. 131.

21 W. Kohn, Phys. Rev. 123, 1242 (1961); for the electron spin resonance (ESR) the lack of the effect of the electron interactions is discussed by Y. Yafet, Solid State Physics, Vol. 14, edited by F. Seitz, and D. Turnbull (Academic Press, New York, 1963), p. 92.

${ }^{22}$ O. V. Dimitrova, cond-mat/0407612 (unpublished).

23 M. I. Dyakonov and V. I. Perel, Zh. Eksp. Teor. Fiz. 60, 1954 (1971) [Sov. Phys. JETP 33, 1053 (1971)]

24 M. A. Khodas, and A. M. Finkel'stein, Phys. Rev. B 68, 155114 (2003).

25 L. D. Landau, and E. M. Lifshitz, Course of Theoretical Physics, Vol. 9, E. M. Lifshitz, and L. P. Pitaevskii, Statistical Physics, Part 2 (Pergamon, Oxford, 1980).

26 Guang-Hong Chen, and M. E. Raikh, Phys. Rev. B 60, 4826 (1999).

27 A. M. Finkel'stein, Z. Phys. B: Condens. Matter 56, 189 (1984).

28 A similar argumentation has been applied by one of the authors in the treatment of disorder, $1 / \tau \ll \epsilon_{F}$, in the disordered Fermi liquid, see A. M. Finkel'stein, Zh. Eksp. Teor Fiz. 84, 168 (1983) [Sov. Phys. JETP 57, 97 (1983)]; Sov. Sci. Rev. A, Phys. 14, 1 (1990).

29 T. Koga, J. Nitta, T. Akazaki, and H. Takayanagi, Phys. Rev. Lett. 89, 046801 (2002).

30 J. B. Miller, D. M. Zumbühl, C. M. Marcus, Y. B. Lyanda- 
Geller, D. Goldhaber-Gordon, K. Campman, and A. C. Gossard, Phys. Rev. Lett. 90, 076807 (2003).

31 C. Schierholz, T. Matsuyama, U. Merkt, and G. Meier, Phys. Rev. B 70, 233311 (2004).

32 J. Luo, H. Munekata, F. F. Fang, and P. J. Stiles, Phys. Rev. B 38, R10142 (1988).

33 B. Das, D. C. Miller, S. Datta, R. Reifenberger, W. P. Hong, P. K. Bhattacharya, J. Singh, and M. Jaffe, Phys. Rev. B 39, 1411 (1989).

34 J. Nitta, T. Akazaki, H. Takayanagi, and T. Enoki, Phys. Rev. Lett. 78, 1335 (1997).

${ }^{35}$ G. Engels, J. Lange, Th. Schäpers, and H. Lüth, Phys. Rev. B 55, R1958 (1997).

36 Y. Sato, T. Kita, S. Gozu, and S. Yamada, J. Appl. Phys. 89, 8017 (2001).
37 D. Stein, K. v. Klitzing, and G. Weimann, Phys. Rev. Lett. 51, 130 (1983).

38 H. L. Stormer, Z. Schlesinger, A. Chang, D. C. Tsui, A. C. Gossard, and W. Wiegmann, Phys. Rev. Lett. 51, 126 (1983).

39 C. -M. Hu, C. Zehnder, Ch. Heyn, and D. Heitmann, Phys. Rev. B 67, 201302 (2003).

${ }^{40}$ H. W. Jiang and E. Yablonovitch, Phys. Rev. B 64, 041307 (2001).

41 S. I. Erlingsson, J. Schliemann, and D. Loss, Phys. Rev. B 71, 035319 (2005).

42 R. Eppenga and M. F. H. Schuurmans, Phys. Rev. B 37, 10923 (1988). 